\title{
Lösungen der Aufgaben
}

\section{Zu Kapitel 1}

1.1. Wir betrachten $\delta: M \rightarrow M$ mit $\delta(x)=a x$. Die Abbildung $\delta$ ist injektiv, denn aus $\delta(x)=\delta(y)$ folgt $x=1 \cdot x=b a x=b \cdot \delta(x)=b \cdot \delta(y)=b a y=1 \cdot y=y$. Da $M$ endlich ist, ist $\delta$ surjektiv. Sei $c \in M$ ein Urbild von $1 \in M$, d. h., es gilt $1=$ $\delta(c)=a c$. Dann ist $b=b \cdot 1=b a c=1 \cdot c=c$ und $a b=1$.

1.2. Die Menge der Abbildungen $f: \mathbb{N} \rightarrow \mathbb{N}$ bildet mit der Hintereinanderausführung von Funktionen ein unendliches Monoid. Das neutrale Element ist die identischen Abbildung id mit id $(n)=n$. Sei $a: \mathbb{N} \rightarrow \mathbb{N}$ mit $a(0)=0$ und $a(n)=n-1$ für $n \geq 1$. Für die Abbildung $b: \mathbb{N} \rightarrow \mathbb{N}$ mit $b(n)=n+1$ gilt $a \circ b=$ id; außerdem zeigt $(b \circ a)(0)=b(a(0))=b(0)=1$, dass $b \circ a \neq$ id gilt.

1.3. Mit $c=b a b$ ist $a c a=a b a b a=a b a=a$ und $c a c=c$.

1.4. Sei $a_{n+1} \in S$ beliebig. Betrachte die $n+1$ Elemente $a_{1} \cdots a_{i}$ für $1 \leq i \leq n+1$. Nach dem Schubfachschluss existieren $1 \leq i<j \leq n+1$ mit $a_{1} \cdots a_{i}=a_{1} \cdots a_{j}$. Insbesondere gilt $i \leq n$. Für $b=a_{i+1} \cdots a_{j}$ gilt die Behauptung.

1.5. Sei $X$ eine endliche Menge mit $1_{U} \notin X$. Dann bildet $U_{X}=X \cup\left\{1_{U}\right\}$ mit der Verknüpfung $a b=b$ für $b \in X$ und $a b=a$ für $b=1_{U}$ ein Monoid mit $|X|+1$ Elementen. Wenn $Y \subseteq X$ gilt, dann ist $U_{Y}$ ein Untermonoid von $U_{X}$. Wählen wir $Y \subseteq X$ mit $|X|$ gerade und $|Y|$ ungerade, so erfüllen $M=U_{X}$ und $U=U_{Y}$ die Forderung der Aufgabe.

1.6. Es ist die Assoziativität nachzuweisen: $\left((x, s, y) \circ\left(x^{\prime}, s^{\prime}, y^{\prime}\right)\right) \circ\left(x^{\prime \prime}, s^{\prime \prime}, y^{\prime \prime}\right)=$ $\left(x, s \varphi\left(y, x^{\prime}\right) s^{\prime}, y^{\prime}\right) \circ\left(x^{\prime \prime}, s^{\prime \prime}, y^{\prime \prime}\right)=\left(x, s \varphi\left(y, x^{\prime}\right) s^{\prime} \varphi\left(y^{\prime}, x^{\prime \prime}\right) s^{\prime \prime}, y^{\prime \prime}\right)=(x, s$, $y) \circ\left(x^{\prime}, s^{\prime} \varphi\left(y^{\prime}, x^{\prime \prime}\right) s^{\prime \prime}, y^{\prime \prime}\right)=(x, s, y) \circ\left(\left(x^{\prime}, s^{\prime}, y^{\prime}\right) \circ\left(x^{\prime \prime}, s^{\prime \prime}, y^{\prime \prime}\right)\right)$.

1.7. (a) Wir weisen zunächst nach, dass jedes linksinverse Element auch rechtsinvers ist. Sei $h$ das linksinverse Element zu $g$ und $g^{\prime}$ das linksinverse Element zu $h$. Es gilt also $h g=e$ und $g^{\prime} h=e$. Dann gilt $g h=e g h=g^{\prime} h g h=g^{\prime} e h=g^{\prime} h=e$. Damit ist $h$ also auch rechtsinvers zu $g$. Außerden gilt somit $g e=g h g=g$

1.7. (b) Sei $|G|>1$ beliebig mit der Verknüpfung $x y=x$ für alle $x, y \in G$. Dann erfüllt jedes Element $e \in G$ die Bedingungen, $G$ ist aber keine Gruppe.

1.7. (c) Sei $a \in G$ fest. Es existiert ein eindeutiges Element $e \in G$ mit $e a=a$. Für alle $b \in G$ gibt es ein $x \in G$ mit $b=a x$. Es ist somit $e b=e a x=a x=b$ und $e$ ist ein linksneutrales Element. Linksinverse Elemente existieren wegen der eindeutigen Lösung von $y \cdot a=e$. Nach Aufgabe 1.7. (a) ist $G$ eine Gruppe.

1.8. (a) (i) $\Rightarrow$ (ii): Es ist $1 \in S$ und damit $S \neq \varnothing$. Da für alle $y \in S$ auch $y^{-1} \in S$ ist, gilt für alle $x, y \in S$ wegen der Abgeschlossenheit, dass $x y^{-1} \in S$ ist. (ii) $\Rightarrow$ (i): Wegen $S \neq \varnothing$ gibt es ein $x \in S$. Damit ist auch $1=x \cdot x^{-1} \in S$ und somit ebenfalls 
$x^{-1}=1 \cdot x^{-1} \in S$. Die Abgeschlossenheit von $S$ folgt mit $x y=x\left(y^{-1}\right)^{-1} \in S$. Also ist $S$ eine Untergruppe von $G$.

1.8. (b) (i) $\Rightarrow$ (ii): Dies gilt wegen der Abgeschlossenheit der Multiplikation und wegen $1 \in S$. (ii) $\Rightarrow$ (i): Sei $x \in S$. Da $S$ endlich ist und $x^{i} \in S$ für alle $i>0$, ist die Ordnung $r$ von $x$ endlich. Also ist $1=x^{r} \in S$ und $x^{-1}=x^{r-1} \in S$ und damit $S$ eine Gruppe.

1.8. (c) Sei $G$ die additive Gruppe $\mathbb{Z}$, und sei $S=\mathbb{N}$. Dann ist $S$ abgeschlossen unter der Addition, aber $S$ ist keine Gruppe.

1.9. Aus $m^{2}=1$ folgt, dass $m$ selbst das Inverse von $m \in M$ ist. Weiter gilt $m n=$ $m \cdot 1 \cdot n=m(m n)(m n) n=m m(n m) n n=1 \cdot n m \cdot 1=n m$ für alle $m, n \in M$. Also ist $M$ eine kommutative Gruppe.

1.10. Sei $U$ die Menge der Elemente mit ungerader Ordnung. Wegen $1 \in U$ ist sie nicht leer. Wir zeigen, dass $U$ eine Untergruppe von $G$ bildet. Seien $x, y \in U$, sei $k$ die Ordnung von $x$, und sei $\ell$ die Ordnung von $y$. Da $G$ kommutativ ist, gilt $\left(x y^{-1}\right)^{k \ell}=$ $x^{k \ell} y^{-k \ell}=\left(x^{k}\right)^{\ell}\left(y^{\ell}\right)^{-1}=1$. Also ist die Ordnung von $x y^{-1}$ ein Teiler von $k \ell$. Da $k \ell$ ungerade ist, ist auch die Ordnung von $x y^{-1}$ ungerade, und es gilt $x y^{-1} \in U$. Mit Aufgabe 1.8. (a) folgt daraus die Behauptung.

1.11. Da $H$ eine Untergruppe ist, gilt $H H=H=H^{-1}$. Aus $x H=y H$ folgt deshalb $x H x^{-1}=x H H x^{-1}=x H H^{-1} x^{-1}=x H(x H)^{-1}=y H(y H)^{-1}=y H H^{-1} y^{-1}=$ $y H H y^{-1}=y H y^{-1}$.

1.12. (a) Sei $g \in G$. Aus $[G: H]=n$ folgt $\left|\left\{g^{i} H \mid i \geq 0\right\}\right| \leq n$. Also existieren $0 \leq j<k \leq n$ mit $g^{j} H=g^{k} H$. Multiplikation mit $g^{-j}$ liefert $H=g^{k-j} H$. Wegen $1 \in H$ folgt daraus $g^{k-j} \in H$ (mit $1 \leq k-j \leq n$ ). Dies beweist die Aussage für $i=k-j$.

1.12. (b) Wir betrachten die Abbildung $\pi: G \rightarrow S_{G / H}$ in die symmetrische Gruppe mit $\pi(g)=\pi_{g}$ und $\pi_{g}$ ist gegeben durch die Abbildungsvorschrift $g^{\prime} H \mapsto g g^{\prime} H$. $\pi$ ist ein Homomorphismus und es ist $\operatorname{ker}(\pi)=N$. Damit ist $N$ ein Normalteiler und $|G / N| \leq n$ ! nach dem Homomorphiesatz. Es verbleibt zu zeigen, dass $N$ der größte Normalteiler ist, der in $H$ enthalten ist. Sei dazu $K \unlhd G$ mit $K \subseteq H$. Dann ist $K=x K x^{-1} \subseteq x H x^{-1}$ für alle $x \in G$. Damit ist auch $K \subseteq \bigcap_{x \in G} x H x^{-1}=N$.

1.13. Sei $D_{4}$ die Bewegungsgruppe eines regelmäßigen Vierecks mit der Eckenmenge $\{0,1,2,3\}$ wie in Abschnitt 1.2. Sie hat acht Elemente und wird von einer Drehung $\delta$ und einer Spiegelung $\sigma$ erzeugt. Ohne Einschränkung lässt $\sigma$ die Ecken 0 und 2 fest. Sei jetzt $\tau$ die Spiegelung, die 1 und 3 fest lässt, so gilt $\delta^{2}=\sigma \tau=\tau \sigma$. Die Untergruppe $K=\langle\sigma, \tau\rangle$ hat den Index 2 in $D_{4}$ und ist damit ein Normalteiler. (Die Gruppe $K$ ist isomorph zur Klein'schen Vierergruppe.) Klar ist auch, dass $\langle\sigma\rangle$ ein Normalteiler in $K$ ist. Schließlich gilt $\delta \sigma \delta^{-1} \neq \sigma$ und damit ist $\langle\sigma\rangle$ kein Normalteiler der $D_{4}$. 
1.14. Sei zunächst $G$ kommutativ. Dann ist $f$ ein Gruppenhomomorphismus aufgrund von $f(x \cdot y)=(x \cdot y)^{-1}=y^{-1} \cdot x^{-1}=x^{-1} \cdot y^{-1}=f(x) \cdot f(y)$. Sei nun $f$ ein Gruppenhomomorphismus. Dann gilt $x \cdot y=\left(y^{-1} \cdot x^{-1}\right)^{-1}=f\left(y^{-1} \cdot x^{-1}\right)=$ $f\left(y^{-1}\right) \cdot f\left(x^{-1}\right)=y \cdot x$. Also ist $G$ kommutativ.

1.15. Sei $n$ die Ordnung von $a$. Dann gilt $f(a)^{n}=f\left(a^{n}\right)=f(1)=1$. Damit ist die Ordnung von $f(a)$ ein Teiler von $n$.

1.16. Sei $0 \neq r \in R$. Dann ist der Homomorphismus $s \rightarrow s r$ injektiv, da $r s=0$ aufgrund der Nullteilerfreiheit $s=0$ nach sich zieht. Aufgrund der Endlichkeit von $R$ ist $s \mapsto s r$ auch surjektiv. Daher gibt es zu $r$ ein Linksinverses $s$ mit $s r=1$. Nach Aufgabe 1.7. (a) ist $(R \backslash\{0\}, \cdot, 1)$ eine Gruppe und damit $R$ mit $1 \neq 0$ ein Schiefkörper.

Bemerkung: Der Satz von Wedderburn (nach Joseph Henry Maclagan Wedderburn, 1882-1942) sagt, dass jeder endliche Schiefkörper schon ein Körper ist, so dass $R$ unter den gegebenen Voraussetzungen sogar kommutativ ist. Ernst Witt (1911-1991) hat Anfang der 1930er Jahre einen Beweis gefunden, der bis heute als der einfachste Beweis für dieses Resultat gilt [1].

1.17. (i) $\Rightarrow$ (ii): Sei $I$ ein Ideal. Dann ist $(I,+, 0)$ ein Normalteiler von $(R,+, 0)$, und nach Satz 1.9 ist $(R / I,+, I)$ eine Gruppe. Weiter gilt für das Produkt der Mengen $r_{1}+I$ und $r_{2}+I$, dass $\left(r_{1}+I\right) \cdot\left(r_{2}+I\right) \subseteq r_{1} r_{2}+r_{1} I+r_{2} I+I \cdot I \subseteq r_{1} r_{2}+I+I+I=r_{1} r_{2}+I$. Sei $r_{1}+I=s_{1}+I$ und $r_{2}+I=s_{2}+I$. Dann existieren $i_{1}, i_{2} \in I$ mit $s_{1}=r_{1}+i_{1}$ und $s_{2}=r_{2}+i_{2}$. Damit erhalten wir $\left(s_{1}+I\right)\left(s_{2}+I\right) \subseteq s_{1} s_{2}+I=\left(r_{1}+i_{1}\right)\left(r_{2}+i_{2}\right)+I=$ $r_{1} r_{2}+r_{1} i_{2}+r_{2} i_{1}+i_{1} i_{2}+I \subseteq r_{1} r_{2}+r_{1} I+r_{2} I+I+I \subseteq r_{1} r_{2} I$. Dies zeigt, dass die Multiplikation durch die Zuordnung $\left(r_{1}+I, r_{2}+I\right) \mapsto r+I$ mit $\left(r_{1}+I\right)\left(r_{2}+I\right) \subseteq r+I$ wohldefiniert ist. Die Assoziativität der Multiplikation und das Distributivgesetz auf $R / I$ folgen nun aus den entsprechenden Gesetzen auf $R$.

(ii) $\Rightarrow$ (iii): Sei $R / I$ ein Ring. Dann ist $\varphi: R \rightarrow R / I: r \mapsto r+I$ nach Satz 1.9 ein Gruppenhomomorphismus bezüglich der Addition mit Kern $I$. Zu zeigen bleibt noch, dass $\varphi$ ein Ringhomomorphismus ist. Es gilt $\varphi(1)=1+I$ und $1+I$ ist das neutrale Element der Multiplikation in $R / I$. Des Weiteren ist $\varphi\left(r_{1}\right) \varphi\left(r_{2}\right)=\left(r_{1}+I\right)\left(r_{2}+\right.$ $I)=r_{1} r_{2}+I=\varphi\left(r_{1} r_{2}\right)$. (iii) $\Rightarrow$ (i): Sei $\varphi: R \rightarrow S$ ein Homomorphismus von kommutativen Ringen mit Kern $I$. Nach Satz 1.9 ist $I$ ein Normalteiler von $R$ und damit insbesondere eine Untergruppe. Für $r \in R$ gilt $\varphi(r I)=\varphi(r) \varphi(I)=\varphi(r) \cdot\{0\}=$ $\{0\}$. Damit ist $r I$ eine Teilmenge des Kerns $I$ von $\varphi$. Dies zeigt, dass $I$ ein Ideal ist.

1.18. Wir betrachten die Abbildung $\varphi: R / I \rightarrow R / J, r+I \mapsto r+J$. Diese ist wohldefiniert und surjektiv da $I \subseteq J$. Da $\left(r_{1}+J\right) \cdot\left(r_{2}+J\right)=r_{1} r_{2}+J$ ist, ist $\varphi$ ein Homomorphismus. Sei $\varphi(r+I)=J$. Dies ist genau dann der Fall, wenn $r+J=J$. Was wiederum genau dann der Fall ist, wenn $r \in J$. Also ist $\operatorname{ker}(\varphi)=J / I$. Die Aussage folgt mit dem Homomorphiesatz.

1.19. (i) Es ist $\left(i_{1}+j_{1}\right)+\left(i_{2}+j_{2}\right)=\left(i_{1}+i_{2}\right)+\left(j_{1}+j_{2}\right) \in I+J$. Anhand dieser Gleichung sieht man direkt, dass $I+J$ eine kommutative Untergruppe ist, da sich die 
Eigenschaften von $I$ und $J$ übertragen. Außerdem gilt $r_{1}(i+j) r_{2}=r_{1} i r_{2}+r_{1} j r_{2} \in$ $I+J$. Also ist $I+J$ ein Ideal. (ii) Man betrachte $I=J=\langle x, y\rangle$ in $R=K[x, y]$ für einen Körper $K$. Dann ist $x^{3}, y^{3} \in I \cdot J$. Falls $I \cdot J$ ein Ideal ist, so muss auch $x^{3}+y^{3} \in I \cdot J$ sein. Wir betrachten allgemein $x^{3}+y^{3}=(a x+b y)(c x+d y)=$ $a c x^{2}+(a d+b c) x y+b d y^{2}$ für $a, b, c, d \in K[x, y]$. Also muss der Grad von $a c$ genau 1 sein. Sei ohne Einschränkung $a=x$. Dann muss $d x+b c=0$ sein. Da jedoch weder $b$ noch $c$ ein $x$ enthalten können, ist dies nicht lösbar. Also ist das (Komplex)Produkt zweier Ideale im Allgemeinen kein Ideal. (iii) Wir betrachten $I=\langle x\rangle$ und $J=\langle y\rangle$ in $R=K[x, y]$. Dann ist $x, y \in I \cup J$, aber nicht $x+y \in I \cup J$. Also ist $I \cup J$ im Allgemeinen kein Ideal. (iv) Der Schnitt zweier Gruppen ist wieder eine Gruppe. Es bleibt also noch $R(I \cap J) R \subseteq I \cap J$ zu zeigen. Sei $x \in I \cap J$. Dann ist $r_{1} x r_{2} \in I$ und $r_{1} x r_{2} \in J$ da $I$ und $J$ Ideale sind. Also ist $r_{1} x r_{2} \in I \cap J$ und somit $I \cap J$ auch ein Ideal.

1.20. Sei $I=\left\langle 6, x^{2}-2\right\rangle$. Wir nehmen an, dass $I=(r(x))$. Dann muss wegen der Gradformel der Grad von $r$ kleiner gleich dem Grad von 6 sein. Also ist der Grad von $r(x)$ genau 0. Da $\pm 1, \pm 2, \pm 3 \notin I$, kann man ohne Einschränkungen $r(x)=6$ annehmen. Dann ist jedoch $x^{2}-2 \notin(r(x))=I$, ein Widerspruch. Der Restklassenring $R / I$ ist kein Körper, da $(2+I)(3+I)=6+I=I$ ist. Damit kann $I$ nicht maximal sein.

1.21. Es ist $3^{4}=81 \equiv 1 \bmod 16$, also ist die Ordnung von 3 ein Teiler von 4 . Wegen $3^{2}=9 \not \equiv 1 \bmod 16$ ist die Ordnung genau 4 .

1.22. Die Gruppe $(\mathbb{Z} / 60 \mathbb{Z})^{*}$ enthält $\varphi(60)=\varphi\left(2^{2}\right) \cdot \varphi(3) \cdot \varphi(5)=16$ Elemente. Die Ordnungen der Elemente sind Teiler von 16. Bis auf die Zahl 1 sind die Teiler gerade. Zur Ordnung 1 gehört nur die Eins, es verbleiben genau 15 Elemente mit gerader Ordnung.

1.23. In $\mathbb{Z} / m n \mathbb{Z}$ existiert ein Element der Ordnung $m n$. In der Gruppe $(\mathbb{Z} / m \mathbb{Z}) \times$ $(\mathbb{Z} / n \mathbb{Z})$ gilt jedoch für die Ordnung $r$ eines jeden Gruppenelements, dass $r$ ein Teiler $\operatorname{von} \operatorname{kgV}(m, n)$ ist. Wegen $\operatorname{ggT}(m, n)>1$ gilt jedoch $\operatorname{kgV}(m, n)=m n / \operatorname{ggT}(m, n)$ $<m n$.

1.24. Nach dem euklidischen Algorithmus gilt: $98=2 \cdot 51-4$ und $51=13 \cdot 4-1$. Rückwärts Einsetzen liefert $1=13 \cdot 4-51=13(2 \cdot 51-98)-51=-13 \cdot 98+25 \cdot 51$. Daraus ergibt sich $s=25$.

1.25. Es gilt $n_{i} \equiv-1 \bmod 3, n_{i} \equiv-1 \bmod 4$ und $n_{i} \equiv-1 \bmod 7$. Dies liefert die möglichen Lösungen $n_{1}=-1+84=83$ und $n_{2}=-1+2 \cdot 84=167$, denn $84=3 \cdot 4 \cdot 7$, und in diesem Bereich gibt es genau eine Lösung.

1.26. Für alle $n \in \mathbb{N}$ ist $n^{4}+n^{2}$ eine gerade Zahl, d.h. $2 n^{4}+2 n^{2}=4 m$. Damit ist $7^{2 n^{4}+2 n^{2}}=7^{4 m}=49^{2 m} \equiv(-11)^{2 m} \equiv 121^{m} \equiv 1^{m} \equiv 1 \bmod 60$. 
1.27. Modulo 6 gilt $X^{2}+X=X(X+1)=(X-2)(X-3)$, und das Polynom hat die Nullstellen $0,2,3,5$. Insbesondere gibt es 4 Nullstellen, obwohl das Polynom nur den Grad 2 hat.

1.28. $z^{4}$

$$
\begin{array}{r}
\frac{-z^{4}+2 z^{3}-2 z^{2}}{2 z^{3}-2 z^{2}} \\
\frac{-2 z^{3}+4 z^{2}-4 z}{2 z^{2}-4 z+4} \\
\frac{-2 z^{2}+4 z-4}{0}
\end{array}
$$

$$
+4=\left(z^{2}-2 z+2\right)\left(z^{2}+2 z+2\right)
$$

Damit ist $p(z)=z^{2}+2 z+2$ und $z^{4}+4=p(-z) \cdot p(z)$. Ableiten ergibt $p^{\prime}(z)=$ $2 z+2$; also hat $p$ ein Minimum bei $z=-1$ und es gilt $p(-1)=1$. Daraus folgt $\forall z \in \mathbb{Z}: p(z) \geq 1$. Damit $z^{4}+4$ eine Primzahl ist, muss also $p(z)=1$ oder $p(-z)=1$ gelten. Dies ist genau bei $z=-1$ bzw. bei $z=1$ der Fall. In beiden Fällen ergibt sich der Wert 5.

1.29. Sei $f=X^{8}+X^{7}+X^{6}+X^{4}+X^{3}+X+1$ und $g=X^{6}+X^{5}+X^{3}+X$. Wir berechnen $\operatorname{ggT}(f, g)$ mithilfe des euklidischen Algorithmus.

$$
\begin{aligned}
f & =g\left(X^{2}+1\right) & & +X^{4}+X^{3}+1 \\
g & =\left(X^{4}+X^{3}+1\right) X^{2} & & +X \\
X^{4}+X^{3}+1 & =X\left(X^{3}+X^{2}\right) & & +1 \\
X & =1 \cdot X & & +0
\end{aligned}
$$

Also ist $\operatorname{ggT}(f, g)=1$

1.30. Angenommen, $f(X)$ ist nicht irreduzibel über $\mathbb{F}_{2}$. Dann gilt $f(X)=g(X) h(X)$ über $\mathbb{F}_{2}$ mit $1 \leq \operatorname{deg}(g), \operatorname{deg}(h) \leq 3$ und $\operatorname{deg}(g)+\operatorname{deg}(h)=5$. Da $f(X)$ keine Nullstelle in $\mathbb{F}_{2}$ hat, muss $2 \leq \operatorname{deg}(g), \operatorname{deg}(h)$ sein. Sei ohne Einschränkung $\operatorname{deg}(g)=2$. Die Polynome vom Grad 2 über $\mathbb{F}_{2}$ sind genau die Polynome $X^{2}+X+1$, $X^{2}+X, X^{2}+1$ und $X^{2}$. Davon ist nur $X^{2}+X+1$ irreduzibel, da die anderen drei eine Nullstelle in $\mathbb{F}_{2}$ haben. Der Divisionsalgorithmus für Polynome liefert $f(x)=$ $\left(X^{3}+X^{2}\right)\left(X^{2}+X+1\right)+1$. Damit ist $X^{2}+X+1$ kein Teiler von $f(X)$, also ist $f(X)$ irreduzibel.

1.31. Für $t=1$ gilt $f(X)=a_{i} X^{i}$ für ein $i \in \mathbb{N}$ und $a_{i} \neq 0$, da $0 \neq f(X)$. Also gibt es keine positiven Nullstellen. Sei daher $t \geq 2$. Allgemein können wir nach Division mit einer geeigneten Potenz $X^{i}$ annehmen, dass $a_{0} \neq 0$ gilt. Dann ist $f^{\prime}(X)$ ein $t-$ 1 dünnes Polynom und hat mit Induktion höchstens $t-2$ Nullstellen. Zwischen je zwei reellen Nullstellen von $f(X)$ liegt (nach dem Satz von Rolle) mindestens eine Nullstelle von $f^{\prime}(X)$. Dies zeigt die Behauptung. 
1.32. (a) Durch Skalierung können wir $\lambda=1$ annehmen, dies macht die Formeln etwas übersichtlicher. Es folgt $g(X)=\sum_{i} b_{i} X^{i}=\sum_{i}\left(a_{i-1}-a_{i}\right) X^{i}$. Ohne Einschränkung gilt weiter $a_{0} \neq 0$. Betrachte einen Index $i-1$, mit $i \geq 1$, nach dem in der Folge $\left(a_{0}, \ldots, a_{d}\right)$ ein Vorzeichenwechsel stattfindet. Es gilt also $a_{i-1} \neq 0$ und für $a_{i-1}<0$ gilt $a_{i} \geq 0$ (bzw. für $a_{i-1}>0$ gilt $a_{i} \leq 0$ ). Hieraus folgt, dass $a_{i-1}$ bei einem Vorzeichenwechsel stets dasselbe Vorzeichen wie $b_{i}$ hat. Nun ist $b_{0}=-a_{0}$, also haben $b_{0}$ und $a_{0}$ ein verschiedenes Vorzeichen, aber es ist $b_{d+1}=a_{d} \neq 0$. Also muss die Anzahl der Vorzeichenwechsel zugenommen haben.

1.32. (b) Es sei $0<\lambda_{1} \leq \cdots \leq \lambda_{k}$ die Folge der positiven reellen Nullstellen mit Vielfachheiten. Dann gilt $f(X)=\left(X-\lambda_{1}\right) \cdots\left(X-\lambda_{k}\right) h(X)$. Nach $k$-facher Anwendung von Aufgabe 1.32. (a) folgt die Behauptung.

1.33. Für $n=0$ ist die Aussage klar. Sie nun $n \geq 1$. Sei $r=\frac{s}{t}$ mit $s, t \in \mathbb{Z}, t \neq 0$ und $\operatorname{ggT}(s, t)=1$. Nun ist

$$
f\left(\frac{s}{t}\right)=0=\frac{s^{n}}{t^{n}}+a_{n-1} \frac{s^{n-1}}{t^{n-1}}+\cdots+a_{0}
$$

Es folgt $s^{n}+a_{n-1} t s^{n-1}+\cdots+a_{0} t^{n}=0$. Also ist $t$ ein Teiler von $s^{n}$ und daher $t= \pm 1$ wegen $\operatorname{ggT}(s, t)=1$. Damit ist $r \in \mathbb{Z}$. Sei ohne Einschränkung $t=1$. Es folgt $s\left(s^{n-1}+a_{n-1} s^{n-2}+\cdots+a_{1}\right)=-a_{0}$, und damit ist $s=r$ ein Teiler von $a_{0}$.

1.34. Sei $f(X)$ irreduzibel über $\mathbb{Z}$. Sei $f(X)=g(X) h(X)$ über $\mathbb{Q}$. Dann gibt es ein $r \in$ $\mathbb{Q}$ und Polynome $g_{1}(X), h_{1}(X) \in \mathbb{Z}[X]$ mit $f(X)=r g_{1}(X) h_{1}(X)$. Ferner können wir $g_{1}(X), h_{1}(X) \in \mathbb{Z}[X]$ so wählen, dass der größte gemeinsame Teiler der Koeffizienten von $g_{1}(X)$ gleich 1 und auch der größte gemeinsame Teiler der Koeffizienten von $h_{1}(X)$ gleich 1 ist. Direktes Nachrechnen zeigt dann, dass der größte gemeinsame Teiler der Koeffizienten von $g_{1}(X) h_{1}(X)$ auch 1 ist. Wegen $\operatorname{ggT}\left(a_{0}, \ldots, a_{n}\right)=1$ muss damit $r= \pm 1$ sein. Ist also $f(X)$ irreduzibel über $\mathbb{Z}$, so auch über $\mathbb{Q}$. Nun müssen wir nur noch zeigen, dass $f(X)$ irreduzibel über $\mathbb{Z}$ ist. Sei $f(X)=g(X) h(X) \mathrm{mit}$ $g(X), h(X) \in \mathbb{Z}[X], g(X)=b_{r} X^{r}+\cdots+b_{0}$ und $h(X)=c_{s} X^{s}+\cdots+c_{0}$. Wir müssen zeigen, dass $r=0$ oder $s=0$. Nach Voraussetzung ist $p$ ein Primteiler von $a_{0}=b_{0} c_{0}$, also von $b_{0}$ oder $c_{0}$, aber nicht von beiden. Es sei $p \mid b_{0}, p \nmid c_{0}$. Wegen $p \nmid a_{n}$ existiert ein kleinster Index $m$ mit $p \nmid b_{m}$, aber $p \mid b_{i}$ für $i<m$. Setzen wir $c_{j}=0$ für $j>s$, so wird $a_{m}=b_{m} c_{0}+\left(b_{m-1} c_{1}+\cdots+b_{0} c_{m}\right)$. Die Primzahl $p$ teilt die Klammer, aber nicht $b_{m} c_{o}$, also auch nicht $a_{m}$. Das bedeutet $m=n$, also $r=n$ und $s=0$.

1.35. (a) Dies folgt direkt aus dem Kriterium von Eisenstein.

1.35. (b) Wir erhalten $f(X+1)=\frac{(X+1)^{p}-1}{X}=X^{p-1}+\left(\begin{array}{c}p \\ 1\end{array}\right) X^{p-2}+\cdots+\left(\begin{array}{c}p \\ p-1\end{array}\right) \in \mathbb{Z}[X]$. Es gilt $p \mid\left(\begin{array}{c}p \\ i\end{array}\right)$ für $1 \leq i \leq p-1$ und $p^{2} \nmid\left(\begin{array}{c}p \\ p-1\end{array}\right)$.

1.36. Die Multiplikation ist mit dieser Erweiterung nicht mehr assoziativ. Insbesondere gilt $(S(X) \cdot(1-X)) \cdot \sum_{i \geq 0} X^{i} \neq S(X) \cdot\left((1-X) \cdot \sum_{i \geq 0} X^{i}\right)$. 
1.37. Da die Operationen so gewählt sind, dass sie der Berechnung in $\mathbb{R}$ entsprechen, ist $\mathbb{Q}[\sqrt{2}]$ ein Unterring von $\mathbb{R}$. Noch zu zeigen ist also, dass das Inverse von $(a+$ $b \sqrt{2}) \neq 0$ in $\mathbb{Q}[\sqrt{2}]$ liegt. Da entweder $a$ oder $b$ nicht 0 sind, ist $a^{2}+2 b^{2}>0$. Es ist

$$
\frac{1}{a+b \sqrt{2}}=\frac{a-b \sqrt{2}}{a^{2}+2 b^{2}}=\frac{a}{a^{2}+2 b^{2}}+\frac{-b}{a^{2}+2 b^{2}} \sqrt{2} \in \mathbb{Q}[\sqrt{2}]
$$

1.38. Die Menge $M=\{g(X) \mid g(X) \in K[X], g(\alpha)=0\}$ besteht nicht nur aus dem Nullpolynom, da es ein $p(X) \in K[X]$ mit $\operatorname{deg}(p) \geq 1$ und $p(\alpha)=0$. Damit gibt es in $M$ ein Polynom $m(X)$ mit minimalem Grad $\geq 1$, dessen Leitkoeffizient 1 ist (da wir den Leitkoeffizienten durch Multiplikation mit einem Element aus $K$ zu 1 normieren können). Wir behaupten, dass $M=\{f(X) m(X) \mid f(X) \in K[X]\}$ gilt. Die Inklusion von rechts nach links ist trivial. Sei $g(X) \in M$. Dann gibt es Polynome $f(X), r(X) \in K[X] \operatorname{mit} g(X)=f(X) m(X)+r(X)$ und $\operatorname{deg}(r)<\operatorname{deg}(m)$. Wegen $g(\alpha)=m(\alpha)=0$ gilt $r(\alpha)=0$. Wegen der Minimalität von $\operatorname{deg}(m)$ ist damit $r(X)$ das Nullpolynom. Dies zeigt $g(X)=f(X) m(X)$ wie behauptet. Außerdem zeigt diese Rechnung, dass $m(X)$ eindeutig ist.

1.39. Sei $a$ ein Quadrat, d.h., es existiert ein $b$ mit $b^{2}=a$, dann ist $a^{(q-1) / 2)}=$ $b^{q-1}=1$. Also ist $a$ eine Nullstelle von $X^{(q-1) / 2}-1$ und daher $X-a$ ein Teiler von $X^{(q-1) / 2}-1$. Sei Umgekehrt $X-a$ ein Teiler, dann ist $a$ eine Nullstelle von $X^{(q-1) / 2}$ -1 . Also ist $a^{(q-1) / 2}=1$. Mit dem Euler-Kriterium (Satz 1.65) folgt, dass $a$ ein Quadrat ist.

\section{Zu Kapitel 2}

2.1. Der Zeit entsprechend kann man davon ausgehen, dass Friedrich der Große an Voltaire auf französisch schrieb. Wir erhalten: „ce soir sous P a cent sous six“. Dies liest sich als „ce soir souper à Sans-Souci“. Friedrich lud also zum Abendessen auf sein Schloss in Potsdam ein, worauf Voltaire mit einem großen „G“ und kleinem „a“ antwortete. Dies bedeutet „G grand a petit“. Er kündigte also mit ,j’ai grand appétit“ seinen Heißhunger an.

2.2. Es ist naheliegend, zu vermuten, dass sich Aufgabe 1.1. anwenden lässt und dass $c_{k}$ und $d_{k}$ zu einander inverse Abbildungen sein müssen. Dies ist jedoch aufgrund der unterschiedlichen Definitions- und Wertebereiche nicht der Fall. Ein einfaches Gegenbeispiel stellen die Verschlüsselungsfunktion $c_{k}:\{1\} \rightarrow\{1,2\}$ mit $c_{k}(1)=1$ und die Entschlüsselungsfunktion $d_{k}:\{1,2\} \rightarrow\{1\}$ mit $d_{k}(1)=d_{k}(2)=1$ dar.

2.3. (a) Wegen $\mathbb{Z} / 77 \mathbb{Z}=\mathbb{Z} / 7 \mathbb{Z} \times \mathbb{Z} / 11 \mathbb{Z}$ folgt $\varphi(n)=6 \cdot 10=60$.

2.3. (b) Der euklidische Algorithmus liefert $s=7$.

2.3. (c) $x \equiv y^{s}=5^{7} \equiv 47 \bmod 77$. 
2.4. Es ist $x=x^{11-2 \cdot 5}$. Mit dem erweiterten euklidischen Algorithmus berechnen zunächst $\left(x^{5}\right)^{-1} \equiv-183 \bmod 551$. Es ergibt sich $x \equiv 429 \cdot(-183) \cdot(-183) \equiv$ $7 \bmod 551$.

2.5. Es gilt $d(c(x))=\left(x^{e} \bmod n\right)^{s} \bmod n \equiv x^{e s} \equiv x^{1+k\left(p_{i}-1\right)} \equiv x \bmod p_{i}$ für eine Zahl $k \in \mathbb{N}$. Mit dem Chinesischen Restsatz folgt $d(c(x)) \equiv x \bmod n$. Mit $x \in$ $\{0, \ldots, n-1\}$ erhalten wir schließlich $d(c(x))=x$.

2.6. (a) Die verschlüsselte Nachricht ist $y \equiv 17^{2} \equiv 36 \bmod n$.

2.6. (b) Sei $n=11 \cdot 23$. Zunächst bestimmen wir $z_{11} \equiv 36^{\frac{11+1}{4}} \equiv 5 \bmod 11$ und $z_{23} \equiv 36^{\frac{23+1}{4}} \equiv 6 \bmod 23$. Mit dem chinesischen Restsatz ergeben sich aus den Forderungen $z \equiv \pm 5 \bmod 11$ und $z \equiv \pm 6 \bmod 23$ die vier Lösungen $z \in\{6,17,236$, $247\}$.

2.6. (c) Die Codierungsfunktion ist auf dem Definitionsbereich injektiv: Angenommen, es existieren $x, \tilde{x} \in 00\{0,1\}^{4} 00$ mit $x>\tilde{x}$ und $x^{2} \equiv \tilde{x}^{2} \bmod 253$. Dann folgt $(x-\tilde{x})(x+\tilde{x}) \equiv 0 \bmod 253$. Nun gibt es zwei Fälle. Entweder ist einer der beiden Faktoren 0 oder je einer ein Vielfaches von 11 bzw. 23. Es gilt $x-\tilde{x} \neq 0$ und $x+\tilde{x} \not \equiv 0 \bmod 253$, da $x+\tilde{x}$ höchstens 120 ist. Es verbleibt der Fall, dass $x-\tilde{x}$ ein Vielfaches von 11 oder 23 ist. Die Zahlen $x$ und $\tilde{x}$ sind beides Vielfache von 4. Wegen $0 \leq \frac{x-\tilde{x}}{4} \leq 15$ kann $x-\tilde{x}$ nur ein Vielfaches von 11 sein, und es muss $\frac{x-\tilde{x}}{4}=11$ gelten. Mit $\frac{x}{4} \leq 15$ folgt daraus $\frac{\tilde{x}}{4} \leq 4$ und $\frac{x+\tilde{x}}{4} \leq 11+2 \cdot 4=19<23$. Insbesondere ist $x+\tilde{x}$ nicht durch 23 teilbar. Dies ist ein Widerspruch.

2.7. (a) Da $n$ eine Primzahl ist, gilt $\varphi(n)=46$ und die Ordnung von $g$ ist ein Teiler von $46=2 \cdot 23$. Es ist $5^{2}=25 \not \equiv 1 \bmod 47$ und $5^{23} \equiv-1 \bmod 47$. Also ist 46 die Ordnung von $g$.

2.7. (b) Es ist $A \equiv 5^{a} \equiv 5^{16} \equiv 17 \bmod 47$ und $B \equiv 5^{b} \equiv 5^{9} \equiv 40 \bmod 47$. Der geheime Schlüssel ist $k=A^{b} \equiv B^{a} \equiv 21 \bmod 47$.

2.7. (c) $B=40$ wurde bereits bestimmt. Der Geheimtext ergibt sich durch $y \equiv A^{b} \cdot x \equiv$ $k \cdot x \equiv 21 \cdot 33 \equiv 35 \bmod 47$.

2.8. Wir lösen das Problem mit dynamischem Programmieren. Dabei füllen wir eine $\{0, \ldots, c\} \times n$ Tabelle $T$. Mit $T_{i, j}$ ist der Eintrag in Zeile $i$, Spalte $j$ gemeint. Die Tabelle wird mit $T_{0, j}=1$ initialisiert. Danach wird sie iterativ durch die folgende Vorschrift gefüllt.

$$
T_{i, j}= \begin{cases}1 & \text { falls } T_{i-s_{j}, j-1}=1 \text { oder } T_{i, j-1}=1 \\ 0 & \text { sonst }\end{cases}
$$

Der Eintrag $T_{i, j}$ bedeutet, dass eine Lösung für das Gewicht $i$ bereits bei Verwendung von $s_{1}, \ldots, s_{j}$ existiert. Eine Lösung des Rucksackproblems existiert also, falls $T_{c, n}=$ 1 ist. 
2.9. Das Inverse von $u$ ist $w=5$ in $(\mathbb{Z} / 71 \mathbb{Z})^{*}$. Alice wählte die $a_{i}$ über $s_{i}=a_{i} \cdot w$ $\bmod 47$. Sie verwendete also die stark wachsende Folge $(2,5,9,17,37)$. Alice bestimmt $c=90 \cdot w=90 \cdot 5 \equiv 24 \bmod 71$. Die einzige Lösung des Rucksackproblems ist $24=1 \cdot 2+1 \cdot 5+0 \cdot 9+1 \cdot 17+0 \cdot 37$. Der Klartext lautet also $(1,1,0,1,0)$.

2.10. Für $i=0$ ist die Aussage trivial und für $i \geq 0$ gilt mit Induktion:

$$
s_{i+1} \geq 2 s_{i}=s_{i}+s_{i}>s_{i}+\sum_{j=1}^{i-1} s_{j}=\sum_{j=1}^{i} s_{j}
$$

2.11. Da jedes Element $x \in X$ ein eindeutiges Bild $y=h(x)$ besitzt, wird jedes $x$ in genau einem $\|y\|$ gezählt. Es folgt $\sum_{y \in Y}\|y\|=|X|$. Setzt man diese Gleichung in $m=\sum_{y \in Y}\|y\| /|Y|$ ein, so ergibt sich direkt $m=\frac{|X|}{|Y|}$. Eine Kollision $\left(x, x^{\prime}\right)$ mit $h(x)=h\left(x^{\prime}\right)=y$ sind je zwei verschiedene Elemente, die bei $\|y\|$ gezählt werden. Die Anzahl dieser Paare für ein festes $y$ ist $\left(\begin{array}{c}\|y\| \\ 2\end{array}\right)$. Damit gilt $N=\sum y \in Y\left(\begin{array}{c}\|y\| \\ 2\end{array}\right)$. Die Gleichung $\sum_{y \in Y}\left(\begin{array}{c}\|y\| \\ 2\end{array}\right)=\frac{1}{2} \sum_{y \in Y}\|y\|^{2}-\frac{|X|}{2}$ ergibt sich mit $\left(\begin{array}{c}\|y\| \\ 2\end{array}\right)=\frac{\|y\|(\|y\|-1)}{2}$ und $\sum_{y \in Y}\|y\|=|X|$. Es ist

$$
\begin{aligned}
& \sum_{y \in Y}(\|y\|-m)^{2}=\sum_{y \in Y}\left(\|y\|^{2}-2\|y\| \frac{|X|}{|Y|}+\left(\frac{|X|}{|Y|}\right)^{2}\right) \\
= & \left(\sum_{y \in Y}\|y\|^{2}\right)-2|X| \frac{|X|}{|Y|}+|Y|\left(\frac{|X|}{|Y|}\right)^{2}=2 N+|X|-\frac{|X|^{2}}{|Y|}
\end{aligned}
$$

Damit gilt insbesondere $0 \leq \sum_{y \in Y}(\|y\|-m)^{2}=2 N+|X|-\frac{|X|^{2}}{|Y|}$ und daraus folgt $N \geq \frac{1}{2}\left(\frac{|X|^{2}}{|Y|}-|X|\right)$.

2.12. Für $i=1$ ist $h_{i}$ kollisionsresistent. Sei nun $i \geq 1$, und sei $x_{1} x_{2} \neq x_{1}^{\prime} x_{2}^{\prime}$ eine Kollision von $h_{i+1}$. Ohne Einschränkung sei $x_{1} \neq x_{1}^{\prime}$. Nach der Definition von $h_{i+1}$ sind zwei Fälle möglich. Entweder ist $h_{i}\left(x_{1}\right) h_{i}\left(x_{2}\right)=h_{i}\left(x_{1}^{\prime}\right) h_{i}\left(x_{2}^{\prime}\right)$ und damit $x_{1} \neq$ $x_{1}^{\prime}$ eine Kollision von $h_{i}$, oder es ist $h_{i}\left(x_{1}\right) h_{i}\left(x_{2}\right) \neq h_{i}\left(x_{1}^{\prime}\right) h_{i}\left(x_{2}^{\prime}\right)$ eine Kollision von $h_{1}$.

2.13. Es gilt $a^{80115359} \equiv a^{1294755} \bmod n$. Also ist $a^{80115359-1294755} \equiv 1 \bmod n$. Es gilt $80115359-1294755=78820604=4 \cdot 19705151$. Da $\varphi(n)=4 p^{\prime} q^{\prime}$ ist, versuchen wir, eine Zahl $b$ zu finden mit $b^{19705151} \not \equiv 1 \bmod n$. Wir wählen zufällig $b=13$. Es ist $13^{19705151} \equiv 10067 \bmod n$ und $10067^{2} \equiv 1 \bmod n$. Wir berechnen $\operatorname{ggT}(10066, n)=719$ und $\operatorname{ggT}(10068, n)=839$. Es ergibt sich somit $n=719 \cdot 839$.

2.14. (a) Sei zunächst $u_{k}(x)=(\gamma, \delta)=\left(\alpha^{s},(x-m \gamma) s^{-1}\right)$. Dann ist $\beta^{\gamma} \gamma^{\delta} \equiv$ $\alpha^{m \gamma} \gamma^{\delta} \equiv \alpha^{m \gamma} \alpha^{s \delta} \equiv \alpha^{m \gamma} \alpha^{s \cdot s^{-1}(x-m \gamma)} \equiv \alpha^{x} \bmod p$, und es folgt $v_{k}(x, \gamma, \delta)=$ true. Sei umgekehrt $v_{k}(x, \gamma, \delta)=$ true und $t$ der diskrete Logarithmus von $\gamma$ zur Basis $\alpha$. Es gilt $\beta^{\gamma} \gamma^{\delta} \equiv \alpha^{x} \bmod p$, und damit ist $\alpha^{x-m \gamma} \equiv \gamma^{\delta} \equiv \alpha^{t \delta} \bmod p$. Es folgt $x-m \gamma \equiv t \delta \bmod (p-1)$. Falls $t$ in $\mathbb{Z} /(p-1) \mathbb{Z}$ invertierbar ist, ist dies äquivalent zu $\delta \equiv(x-m \gamma) t^{-1} \bmod (p-1)$. Folglich ist $(\gamma, \delta)$ eine gültige Unterschrift für $x$. 
2.14. (b) Wähle $u, v$ mit $\operatorname{ggT}(v, p-1)=1$. Wir setzen $\gamma=\alpha^{u} \beta^{v} \bmod p, \delta=$ $-\gamma v^{-1} \bmod p-1$ und $x=u \delta \bmod p-1$. Damit ist dann $\beta^{\gamma} \gamma^{\delta}$ $\equiv \beta^{\gamma} \alpha^{u \delta} \beta^{-v v^{-1} \gamma} \equiv \alpha^{u \delta} \equiv \alpha^{x} \bmod p$.

2.14. (c) Es ist zu zeigen, dass $\beta^{\lambda} \lambda^{\mu} \equiv \alpha^{x^{\prime}} \bmod p$ ist. Wir setzen $y=(h \gamma-j \delta)^{-1}$ $\bmod p-1$. Da $(\gamma, \delta)$ eine gültige Unterschrift ist, gilt $\gamma^{\delta} \equiv \beta^{-\gamma} \alpha^{x} \bmod p$. Damit folgt $\beta^{\lambda} \lambda^{\mu} \equiv \beta^{\lambda}\left(\gamma^{h} \alpha^{i} \beta^{j}\right)^{\delta \lambda y} \equiv \beta^{\lambda}\left(\gamma^{\delta}\right)^{h \lambda y} \alpha^{i \delta \lambda y} \beta^{j \delta \lambda y} \equiv \beta^{\lambda} \beta^{-\gamma h \lambda y} \alpha^{x h \lambda y} \alpha^{i \delta \lambda y}$ $\beta^{j \delta \lambda y} \equiv \beta^{\lambda} \beta^{-\gamma h \lambda y} \alpha^{x^{\prime}} \beta^{j \delta \lambda y} \equiv \alpha^{x^{\prime}} \beta^{\lambda-\lambda(h y-j \delta)(h y-j \delta)^{-1}} \equiv \alpha^{x^{\prime}} \bmod p$.

2.15. Wir wählen die Primzahl $p=61$. Mithilfe des Polynoms $a(X)=42+a_{1} X \in$ $\mathbb{F}_{p}[X]$ wollen wir das Geheimnis aufteilen. Dazu wählen wir zufällig $a_{1}=23$. Es ergibt sich $a(1)=4, a(2)=27$ und $a(3)=50$. Damit ergeben sich die Informationen $(1,4),(2,27)$ und $(3,50)$. Wir zeigen exemplarisch, dass mit zwei dieser Informationen das Geheimnis rekonstruiert werden kann.

Seien die Informationen $(1,4)$ und $(2,27)$ gegeben. Es ergeben sich die Gleichungen $a_{0}+a_{1}=4$ und $a_{0}+2 a_{1}=27$. Wir lösen die erste Gleichung nach $a_{0}$ auf und setzen das Ergebnis in die zweite Gleichung ein. Es ergibt sich $\left(4-a_{1}\right)+2 a_{1}=$ 27 , also $a_{1}=27-4=23$. Damit gilt $a_{0}+23=4$, also $a_{0} \equiv-19 \equiv 42 \bmod 61$.

2.16. Wir benutzen ein mehrstufiges Verfahren auf der Basis des Shamir-Verfahrens. Dazu wird der geheime Schlüssel zunächst einmal so auf drei Schlüssel verteilt, dass zwei davon ausreichen um den geheimen Schlüssel zu bekommen. Dann werden zwei dieser Schlüssel an die Direktoren verteilt. Der dritte Schlüssel wird wieder in zehn Schlüssel aufgeteilt, wobei sieben dieser Schlüssel ausreichen um das Geheimnis zu entschlüsseln. Dann werden sieben dieser zehn Schlüssel an die Abteilungsleiter weitergegeben. Die restlichen drei Schlüssel werden wieder als Geheimnis aufgeteilt (dazu benötigt man eine geeignete Codierung der drei Schlüssel in ein Geheimnis). Dieses mal wird das Geheimnis der drei Schlüssel aufgeteilt in insgesamt 87 Schlüssel, wobei elf davon ausreichen um das Geheimnis zu rekonstruieren. Also können elf Mitarbeiter die fehlenden drei Abteilungsleiter ausgleichen und sieben Abteilungsleiter können einen fehlenden Direktor ausgleichen.

2.17. Die Personen 1,2 und 3 haben Gehälter $g_{1}, g_{2}$ und $g_{3}$. Das Protokoll funktioniert folgendermaßen: Zunächst schickt die Person 1 eine zufällige Zahl $z$ an Person 2. Diese schickt dann die Zahl $z+g_{2}$ an Person 3. Da Person 3 die Zufallszahl $z$ nicht kennt, kann diese daraus nicht das Gehalt $g_{2}$ berechnen. Dann schickt Person 3 die Summe $z+g_{2}+g_{3}$ an Person 1. Person 1 kann daraus, da sie die Zahl $z$ kennt, die Summe $g_{1}+g_{2}+g_{3}$ bilden und somit das Durchschnittsgehalt berechnen. Dieses teilt sie den weiteren beiden Personen mit. Dieses Protokoll lässt sich leicht auf mehr als drei Mitarbeiter verallgemeinern.

2.18. Seien $w_{1}<\ldots<w_{n}$ die möglichen Gehälter. Sei $c_{B}$ Bob’s öffentliche Verschlüsselungsfunktion und $d_{B}$ seine private Entschlüsselungsfunktion. Alice wählt ein zufälliges $x$ und sendet $d=c_{B}(x)-a$ an Bob, wobei $a$ ihr Gehalt ist. Bob berechnet nun $y_{1}, \ldots, y_{n}$ mit $y_{i}=d_{B}\left(d+w_{i}\right)$. Mit $w_{j}=a$ gilt $y_{j}=x$. Um sein Gehalt 
zu verschleiern wendet Bob eine Einwegfunktion $f$ an und berechnet $z_{i}=f\left(y_{i}\right)$. Sei ohne Einschränkung $z_{i} \neq z_{j}+1$ für $1 \leq i, j \leq n$ (andernfalls muss Alice ein neues $x$ wählen oder Alice und Bob einigen sich auf eine andere Hashfunktion). Ist $b=w_{k}$ das Gehalt von Bob, so sendet Bob die Folge $z_{1}, \ldots, z_{k}, z_{k}+1, \ldots, z_{n}+1$ an Alice. Nun ist $a \leq b$ genau dann, wenn $f(x)$ in der Folge vorkommt.

2.19. Der Dealer verpflichtet sich zu einer Zahl zwischen 0 und 36 . Dann geben alle Spieler Ihre Gebote in Klartext ab. Der Dealer legt dann seine Zahl offen.

\section{Zu Kapitel 3}

3.1. Sei $\gamma_{0}>0$ so, dass $f(n) \leq \sum_{i=0}^{k} f\left(\left\lceil\alpha_{i} n\right\rceil\right)+\gamma_{0} n$. Weiter sei $\varepsilon>0$ und $n_{0}>0$ so, dass $\alpha_{i} n_{0} \leq n_{0}-1$ für alle $i \in\{1, \ldots, k\}$ und $\sum_{i=0}^{k}\left\lceil\alpha_{i} n\right\rceil \leq(1-\varepsilon) n$ für alle $n \geq n_{0}$ gilt. Wähle schließlich ein $\gamma$ so groß, dass $\gamma_{0}<\gamma \varepsilon$ und $f(n)<\gamma n$ für alle $n<n_{0}$. Mit Induktion nach $n$ zeigen wir jetzt $f(n)<\gamma n$. Für $n<n_{0}$ ist die Behauptung aufgrund der Wahl von $\gamma$ erfüllt. Für $n \geq n_{0}$ gilt:

$$
\begin{aligned}
f(n) & \leq \sum_{i=0}^{k} f\left(\left\lceil\alpha_{i} n\right\rceil\right)+\gamma_{0} n \leq \sum_{i=0}^{k} \gamma \cdot\left\lceil\alpha_{i} n\right\rceil+\gamma_{0} n \\
& \leq\left(\gamma(1-\varepsilon)+\gamma_{0}\right) \cdot n \leq \gamma n
\end{aligned}
$$

Bemerkung: Eine bekannte Anwendung des Master-Theorems II ist der Beweis, dass sich der Median einer Folge von $n$ Zahlen mit nur $\mathcal{O}(n)$ Vergleichen bestimmen lässt. Wir müssen die Folge also nicht erst sortieren, um den Median zu bestimmen.

3.2. Ohne Einschränkung gilt $a \neq 0 \neq b$. Wir teilen $a$ und $b \operatorname{durch} \operatorname{ggT}(a, b)$. Danach können wir die Wurzeln für Zähler und Nenner unabhängig durch binäre Suche bestimmen.

3.3. Es gilt $2^{(n-1) / 2}=2^{864} \equiv 1 \bmod 1729$ und nach Satz 1.67 (c) gilt $\left(\frac{2}{n}\right)=$ $(-1)^{\left(n^{2}-1\right) / 8}=1$. Man beachte, dass $1729 \equiv 1 \bmod 16$ und damit $\frac{n^{2}-1}{8}$ gerade ist. Damit ist 1729 eine Euler'sche Pseudoprimzahl zur Basis 2.

Wir schreiben $1728=2^{\ell} u=2^{6} 27$ und setzen $b=645 \equiv 2^{27} \not \equiv 1 \mathrm{mod}$ 1729. Damit erhalten wir $\left(b^{2^{0}}, b^{2^{1}}, b^{2^{2}}, b^{2^{3}}, b^{2^{4}}, b^{2^{5}}\right)=(645,1065,1,1,1,1)$ modulo 1729. Da -1 in dieser Folge nicht vorkommt, ist 1729 keine starke Pseudoprimzahl zur Basis 2. Außerdem sehen wir mit $c=1065$, dass $c^{2} \equiv 1 \bmod 1729$ gilt. Es folgt $1064 \cdot 1066=(c-1)(c+1) \equiv 0 \bmod 1729$. Damit ist $\operatorname{ggT}(1064,1729)=133$ ein nichttrivialer Teiler von $1729=133 \cdot 13=7 \cdot 13 \cdot 19$.

Wir halten darüber hinaus fest, dass in diesem speziellen Fall von allen $a \in$ $\{1, \ldots, n-1\}$ (bzw. von allen zu $n$ teilerfremden $a \in\{1, \ldots, n-1\}$ ) der Fermat-Test bei 75\% (bzw. 100\%), der Solovay-Strassen-Test bei rund 39,5\% (bzw. rund 52,7\%) und der Miller-Rabin-Test bei rund 9,4\% (bzw. 12,5\%) fehlschlägt. Insbesondere ist 1729 also eine Carmichael-Zahl.

3.4. Sei $r$ die Ordnung von $a$ in $(\mathbb{Z} / n \mathbb{Z})^{*}$. Aus (i) folgt $r \mid n-1$, und mit (ii) ergibt sich schließlich $r=n-1$. Damit gilt $\left|(\mathbb{Z} / n \mathbb{Z})^{*}\right|=n-1$, und $n$ ist eine Primzahl. 
3.5. Angenommen $f_{n}$ ist eine Primzahl. Aus dem Euler-Kriterium folgt $3^{\left(f_{n}-1\right) / 2} \equiv$ $\left(\frac{3}{f_{n}}\right) \bmod f_{n}$. Zusammen mit $f_{n} \equiv 1 \bmod 4$ ergibt sich aus dem quadratischen Reziprozitätsgesetz die Rechnung $\left(\frac{3}{f_{n}}\right)=\left(\frac{f_{n}}{3}\right) \equiv f_{n}^{(3-1) / 2}=f_{n} \equiv-1 \bmod 3$. Die Umkehrung folgt mit dem Lucas-Test in Aufgabe 3.4.

3.6. Wir setzen $f(X)=X^{2}-4 X+1$. Sei zunächst die Kongruenz erfüllt, und sei $q$ der kleinste Primteiler von $n$. In $\mathbb{F}_{q}[X]$ gilt $X^{n+1} \equiv 1 \bmod f(X)$ und $X^{(n+1) / 2} \not \equiv$ $1 \bmod f(X)$. Also hat $X$ in der Einheitengruppe von $R=\mathbb{F}_{q}[X] / f$ die Ordnung $n+$ 1. Wenn $n$ zusammengesetzt ist, dann gilt $q \leq \sqrt{n}$. Da $R$ genau $q^{2}$ Elemente enthält, ist $n \geq q^{2}>\left|R^{*}\right| \geq n+1$. Dies ist ein Widerspruch. Also ist $n$ eine Primzahl.

Für die Umkehrung sei nun $n$ eine Primzahl. Nach dem quadratischen Reziprozitätsgesetz gilt $\left(\frac{3}{n}\right)=-\left(\frac{n}{3}\right)=-\left(\frac{1}{3}\right)=-1$, da $2^{p}-1 \equiv(-1)^{p}-1 \equiv 1 \bmod 3$ für $p$ ungerade. Mit der Mitternachtsformel für quadratische Gleichungen folgt, dass $f$ irreduzibel ist. Also ist $K=\mathbb{F}_{n}[X] / f$ ein Körper. In $\mathbb{F}_{n}$ und damit auch in $K$ gilt $2^{(n-1) / 2}=1$ nach dem Euler-Kriterium, denn es ist $\left(\frac{2}{n}\right)=1$. Außerdem gilt $(X-1)^{2}=2 X$ in $K$. Das Polynom $g(Y)=Y^{2}-4 Y+1$ in $K[Y]$ hat die Nullstellen $X$ und $4-X$. Da $K$ ein Körper ist, sind dies die einzigen Nullstellen. Die Koeffizienten von $g$ sind in $\mathbb{F}_{n}$, so dass $0=g(X)^{n}=g\left(X^{n}\right)$ gilt. Also ist $X^{n} \in\{X, 4-X\}$. Da $Y^{n}-Y$ genau die Elemente aus $\mathbb{F}_{q}$ als Nullstellen besitzt und $X \notin \mathbb{F}_{q}$ gilt, ist $X^{n} \neq X$ und damit $X^{n}=4-X$. Wir berechnen nun $(X-1)^{n+1}$ auf zwei Arten:

$$
\begin{aligned}
(X-1)^{n+1} & =\left((X-1)^{2}\right)^{(n+1) / 2}=(2 X)^{(n+1) / 2}=2 X^{(n+1) / 2} \\
(X-1)^{n+1} & =(X-1)^{n}(X-1)=\left(X^{n}-1\right)(X-1) \\
& =(3-X)(X-1)=-X^{2}+4 X-3=-2
\end{aligned}
$$

Wenn wir diese beiden Rechnungen kombinieren, dann erhalten wir wie gewünscht $X^{(n+1) / 2}=1$ in $K$.

3.7. Sei $R=\mathbb{Z} / n \mathbb{Z}, f(X)=X^{2}-4 X+1$ und $K=R[X] / f$. Mit Induktion nach $j$ zeigen wir zunächst, dass in $K$ die Eigenschaft $\ell_{j}=X^{2^{j}}+(4-X)^{2^{j}}$ gilt. Für $j=0$ ist $\ell_{0}=4=X+(4-X)$. Sei nun $j \geq 0$. Mit $X(4-X)=1$ ergibt sich

$$
\begin{aligned}
\ell_{j+1} & =\ell_{j}^{2}-2=\left(X^{2^{j}}+(4-X)^{2^{j}}\right)^{2}-2 \\
& =X^{2^{j+1}}+(4-X)^{2^{j+1}}+2 X^{2^{j}}(4-X)^{2^{j}}-2 \\
& =X^{2^{j+1}}+(4-X)^{2^{j+1}}+2(X(4-X))^{2^{j}}-2 \\
& =X^{2^{j+1}}+(4-X)^{2^{j+1}}+2 \cdot 1^{2^{j}}-2=X^{2^{j+1}}+(4-X)^{2^{j+1}}
\end{aligned}
$$

Es gilt genau dann $X^{(n+1) / 2}=-1$, wenn $X^{(n+1) / 2-k}=-X^{-k}$ ist. Für $k=(n+1) / 4$ folgt zusammen mit $X^{-1}=4-X$ die Äquivalenz

$$
X^{(n+1) / 2}=-1 \Leftrightarrow \underbrace{X^{(n+1) / 4}+(4-X)^{(n+1) / 4}}_{\ell_{p-2}}=0
$$

Die Behauptung folgt nun aus Aufgabe 3.6. 
3.8. Für $B=\{2,3\}$ ergibt sich $k=2^{7} \cdot 3^{5}=128 \cdot 243=31104$. Mit schneller modularer Exponentiation und $a=2$ ergibt sich $a^{k} \equiv 82 \bmod n$. Es ist $\operatorname{ggT}\left(a^{k}-\right.$ $1, n)=\operatorname{ggT}(81, n)=1$ und wir haben keinen Teiler gefunden.

Für $B=\{2,3,5\}$ ergibt sich $k=2^{7} \cdot 3^{5} \cdot 5^{3}=128 \cdot 243 \cdot 125=3888000$. Mit schneller modularer Exponentiation und $a=2$ ergibt sich $a^{k} \equiv 133 \bmod n$. Damit ist $\operatorname{ggT}\left(a^{k}-1, n\right)=\operatorname{ggT}(132, n)=11$ und wir haben einen nichttrivialen Teiler von $n$ gefunden.

Wir merken darüber hinaus an, dass die ( $p-1)$-Methode für $B=\{2,3\}$ bei rund $24 \%$ und für $B=\{2,3,5\}$ bei rund $84 \%$ aller $a \in\{2, \ldots, n-1\}$ einen nichttrivialen Teiler findet.

3.9. Wir setzen $x_{0}=y_{0}=12$ und erhalten folgende Werte

$$
\begin{array}{lll}
x_{1}=145 & y_{1}=356 & \operatorname{ggT}\left(y_{1}-x_{1}, n\right)=1 \\
x_{2}=356 & y_{2}=144 & \operatorname{ggT}\left(y_{2}-x_{2}, n\right)=53
\end{array}
$$

Damit ist 53 ein Teiler von $n$.

3.10. Die Ordnung $n$ von $(\mathbb{Z} / 19 \mathbb{Z}) *$ ist 18. Wir setzen $g=2, y=3$ und $m=\lfloor\sqrt{n}\rfloor=$ 4. Die Berechnung der Babysteps ergibt folgende Tabelle $B$ :

\begin{tabular}{ccccc}
\hline $\boldsymbol{r}$ & $\mathbf{3}$ & $\mathbf{2}$ & $\mathbf{1}$ & $\mathbf{0}$ \\
\hline$y g^{n-r} \bmod 19$ & 17 & 15 & 11 & 3 \\
\hline
\end{tabular}

Nun kommen die Giantsteps. Wir berechnen $h=2^{4}=16$ und sukzessive $h^{0}=1$, $h^{1}=16, h^{2} \equiv 9$. Keiner dieser Werte findet sich in der zweiten Zeile der Tabelle wieder. Schließlich finden wir für $s=3$ den Wert $h^{s} \equiv 11$ in der Tabelle $B$ und erhalten $r=1$. Damit ist $x=3 \cdot m+r=13$ der gesuchte Wert.

3.11. Man berechnet das kleinste $n \geq 0$ mit $g^{n}=1$. Hierzu testet man die Giantsteps $s$ in aufsteigender Reihenfolge und verwirft die Lösung $r=0$ und $s=0$. Eine kleine Optimierung ergibt sich, wenn wir bei zwei Babysteps $(r, a)$ und $\left(r^{\prime}, a\right)$ mit $r<r^{\prime}$ nur den Eintrag $(r, a)$ in der Tabelle $B$ speichern.

3.12. (a) Die Ordnung von $G$ ist $22=2 \cdot 11$. Es gilt $g^{2}=9 \not \equiv 1 \bmod 23$ sowie $g^{11} \equiv$ $1 \bmod 23$. Damit ist $q=11$ die Ordnung von $g$.

3.12. (b) Wir setzen $y=18$ sowie $f: \mathbb{Z} / q \mathbb{Z} \times \mathbb{Z} / q \mathbb{Z} \rightarrow \mathbb{Z} / q \mathbb{Z} \times \mathbb{Z} / q \mathbb{Z}$ mit

$$
f(r, s)= \begin{cases}(r+1, s) & \text { falls }\left(g^{r} y^{s} \bmod 23\right) \equiv 0 \bmod 3 \\ (2 r, 2 s) & \text { falls }\left(g^{r} y^{s} \bmod 23\right) \equiv 1 \bmod 3 \\ (r, s+1) & \text { falls }\left(g^{r} y^{s} \bmod 23\right) \equiv 2 \bmod 3\end{cases}
$$

und definieren eine Folge $\left(r_{i}, s_{i}\right)$ mit $\left(r_{1}, s_{1}\right)=(1,1)$ und $\left(r_{i+1}, s_{i+1}\right)=f\left(r_{i}, s_{i}\right)$ für $i \geq 1$. Weiterhin setzen wir $h_{i}=g^{r_{i}} y^{s_{i}} \bmod 23$. Für $\ell \in\{1,2,4\}$ berechnen wir die 
Werte von $\left(r_{\ell}, s_{\ell}\right)$ sowie $h_{\ell}$ und für $k \in\{1, \ldots, \ell\}$ wie Werte von $\left(r_{\ell+k}, s_{\ell+k}\right)$ sowie $h_{\ell+k}$ wie in folgender Tabelle dargestellt:

\begin{tabular}{lccccccc}
\hline $\boldsymbol{\ell}$ & $\boldsymbol{\ell}=\mathbf{1}$ & $\boldsymbol{\ell}=\mathbf{2}$ & \multicolumn{2}{c}{$\boldsymbol{\ell = 4}$} \\
\hline$\left(\boldsymbol{r}_{\ell}, s_{\ell}\right)$ & $(1,1)$ & $(1,2)$ & \multicolumn{2}{c}{$(3,2)$} \\
$h_{\ell}$ & 8 & 6 & & \multicolumn{2}{c}{8} & \\
\hline$k$ & $k=1$ & $k=1$ & $k=2$ & $k=1$ & $k=2$ & $k=3$ & $k=4$ \\
\hline$\left(r_{\ell+k}, s_{\ell+k}\right)$ & $(1,2)$ & $(2,2)$ & $(3,2)$ & $(3,3)$ & $(4,3)$ & $(5,3)$ & $(5,4)$ \\
$h_{\ell+k}$ & 6 & 18 & 8 & 6 & 18 & 8 & 6 \\
\hline
\end{tabular}

Der Algorithmus endet falls $h_{\ell}=h_{\ell+k}$, hier also für $\ell=4$ und $k=3$; die Werte für $\ell=k=4$ werden nicht mehr berechnet. Es gilt $g^{3} y^{2}=h_{\ell}=h_{\ell+k}=g^{5} y^{3}$. Falls $y=g^{x}$ ist, dann folgt $g^{3+2 x}=g^{5+3 x}$ und daher $3+2 x \equiv 5+3 x \bmod 11$. Eine Lösung dieser Kongruenz ist 9 . Weiterhin gilt $3^{9} \equiv 18 \bmod 23$ und der gesuchte Wert ist 9. Man beachte, dass der Algorithmus die Übereinstimmung mit Wert 6 von $h_{2}$ und $h_{5}$ nicht findet, da die Werte $h_{5}, h_{6}, h_{7}, h_{8}$ ausschließlich mit $h_{4}$ verglichen werden.

3.13. Wir setzen $g=2$ und $y=5$. Die Ordnung von $(\mathbb{Z} / 19 \mathbb{Z})^{*}$ ist $n=18=2 \cdot 3^{2}$. Wir setzen weiterhin Parameter wie in folgender Tabelle vorgegeben:

\begin{tabular}{cccccc}
\hline $\boldsymbol{p}$ & $\boldsymbol{e}_{\boldsymbol{p}}$ & $\boldsymbol{n}_{\boldsymbol{p}}$ & $\boldsymbol{g}_{\boldsymbol{p}}$ & $\boldsymbol{y}_{\boldsymbol{p}}$ & $\boldsymbol{x}_{\boldsymbol{p}}$ \\
\hline 2 & 1 & 9 & 18 & 1 & 0 \\
3 & 2 & 2 & 4 & 6 & 7 \\
\hline
\end{tabular}

Für $p \in\{2,3\}$ gilt nun $n_{p}=n / p^{e(p)}, g_{p}=g^{n_{p}} \bmod q$ und $y_{p} \equiv y^{n_{p}} \equiv g_{p}^{x_{p}}$ $\bmod q$. Setzen wir $x=16$, so sind die Kongruenzen $x \equiv 0 \bmod 2$ und $x \equiv 7$ mod $3^{2}$ erfüllt und nach Satz 3.10 ist 16 der gesuchte Wert.

Um den Wert $x_{3}$ zu bestimmen machen wir den Ansatz $x_{3}=d_{0}+d_{1} \cdot 3$. Die Stelle $d_{0}$ ergibt sich als Lösung der Gleichung $\left(\left(g_{3}\right)^{3}\right)^{d_{0}} \equiv\left(y_{3}\right)^{3} \bmod 19$. Nun ist $\left(g_{3}\right)^{3}=4^{3} \equiv 7 \bmod 19$ sowie $\left(y_{3}\right)^{3}=6^{3} \equiv 7 \bmod 19$ und $d_{0}=1$ ist trivialerweise eine Lösung. Für die Bestimmung von $d_{1}$ setzen wir zunächst $z_{1}=11$. Damit gilt $z_{1} \equiv y_{3} g_{3}^{-d_{0}} \bmod 19$ und $d_{1}$ ist Lösung der Kongruenz $\left(\left(g_{3}\right)^{3}\right)^{d_{1}} \equiv z_{1} \bmod 19$, d. h., nach Einsetzen ist $7^{d_{1}} \equiv 11 \bmod 19$. Eine Lösung hiervon ist $d_{1}=2$ und wir erhalten $x_{3}=1+2 \cdot 3=7$.

3.14. Die Ordnung von $(\mathbb{Z} / p \mathbb{Z})^{*}$ ist eine Zweierpotenz. Mit der Reduktion der Gruppenordnung nach Pohlig-Hellman genügt es, $\mathcal{O}(\log p)$ diskrete Logarithmen in zweielementigen Gruppen zu berechnen.

3.15. Es gilt die Äquivalenz $a_{n+1} \in a_{1} \mathbb{Z}+\cdots+a_{n} \mathbb{Z} \Leftrightarrow \operatorname{ggT}\left(a_{1}, \ldots, a_{n}\right) \mid a_{n+1}$. Hierbei ist $g=\operatorname{ggT}\left(a_{1}, \ldots, a_{n}\right)=\operatorname{ggT}\left(\operatorname{ggT}\left(a_{1}, \ldots, a_{n-1}\right), a_{n}\right)$. Insbesondere las- 
sen sich dadurch mit dem erweiterten euklidischen Algorithmus Zahlen $y_{i} \in \mathbb{Z}$ mit $a_{1} y_{1}+\cdots+a_{n} y_{n}=g$ berechnen. Wenn wir $x_{i}=y_{i} a_{n+1} / g$ setzen, dann liefert dies eine Lösung für die Gleichung.

3.16. Wir setzen $q=41, u=5$ und $\ell=3$. Damit gilt $q-1=u 2^{\ell}$. Außerdem sehen wir an $g^{(q-1) / 2} \equiv-1 \bmod 41$, dass $g$ kein Quadrat in $\mathbb{F}_{41}$ ist. Im zweiten Schritt bestimmen wir nun sukzessive die Bits $k_{0}, k_{1}$ und $k_{2}$. Sei $x_{i}=a g^{-\sum_{j=0}^{i} k_{j} 2^{j}}$ und insbesondere $x_{-1}=a$. Wir bestimmen $k_{i} \in\{0,1\}$ aus $k_{0}, \ldots, k_{i-1}$ und setzen $k_{i}=0$ genau dann, wenn $x_{i-1}^{u 2^{\ell-i-1}} \equiv 1 \bmod q$ gilt.

- Für $i=0$ gilt $x_{-1}^{u 2^{\ell-1}}=a^{(q-1) / 2} \equiv 1 \bmod q$ und wir setzen $k_{0}=0$. Man beachte, dass die Eingabe nach dem Euler-Kriterium kein Quadrat wäre, wenn wir $k_{0}$ auf 1 setzen würden.

- Für $i=1$ berechnen wir $x_{0}=x_{-1} \cdot g^{k_{0} 2^{0}}=x_{-1}=2$. Damit gilt $x_{0}^{u 2^{\ell-2}} \equiv$ $-1 \bmod q$ und wir setzen $k_{1}=1$.

- Für $i=2$ berechnen wir $x_{1}=x_{0} \cdot\left(g^{-1}\right)^{k_{1} 2^{1}} \equiv 2 \cdot 14^{2} \equiv 23 \bmod 41$; für die zweite Kongruenz benutzen wir $g^{-1} \equiv 14 \bmod 41$. Damit gilt $x_{1}^{u 2^{\ell-3}} \equiv-1 \bmod$ $q$ und wir setzen $k_{2}=1$.

Damit erhalten wir $k=k_{0}+2 k_{1}+4 k_{2}=6$. Schließlich ist $b=2^{(u+1) / 2} g^{-u k / 2}=$ $2^{3} 14^{5 \cdot 3} \equiv 24 \bmod 41$ eine Wurzel von 2 in $\mathbb{F}_{41}$. Die zweite Wurzel ist $17=41-24$.

3.17. Sei zunächst $a^{(p-1) / 4}=1$ und $b=a^{(p+3) / 8}$. Dann ist $b^{2}=a^{(p+3) / 4}=a$. $a^{(p-1) / 4}=a \cdot 1=a$. Sei nun ${ }^{(p-1) / 4}=1$ und $b=2^{-1}(4 a)^{(p+3) / 8}$. Nach dem Euler-Kriterium und dem quadratischen Reziprozitätsgesetz gilt $2^{(p-1) / 2}=\left(\frac{2}{p}\right)=$ -1 . Damit ist $b^{2}=2^{-2}(4 a)^{(p+3) / 4}=2^{-2} \cdot 2^{(p+3) / 2} \cdot a^{(p+3) / 4}=2^{(p-1) / 2} \cdot a^{(p-1) / 4}$. $a=(-1) \cdot(-1) \cdot a=a$.

3.18. (a) Aus dem quadratischen Reziprozitätsgesetz folgt $\left(\frac{-1}{n}\right)=-1$, also ist -1 ein Quadrat und $X^{2}=-1$ besitzt keine Lösung in $\mathbb{F}_{p}$. Da $f(X)$ den Grad 2 hat, ist $f(X)$ irreduzibel.

3.18. (b) Es gilt $\left(\frac{1}{p}\right)=1$ und $\left(\frac{p-1}{p}\right)=\left(\frac{-1}{p}\right)=-1$. Seien daher allgemeiner $1 \leq b<$ $c \leq p-1$ mit $\left(\frac{b}{p}\right)=1$ und $\left(\frac{c}{p}\right)=-1$. Wenn $c=b+1$ gilt, dann setzen wir $a=b$. Andernfalls betrachten wir $d=\lfloor(b+c) / 2\rfloor$ und berechnen $\left(\frac{d}{p}\right)$. Abhängig davon, ob das Ergebnis hiervon -1 oder 1 ist, setzen wir den Algorithmus rekursiv mit den Zahlen $b<d$ oder mit $d<c$ fort. Diese binäre Suche liefert die gesuchte Zahl $a$ nach höchstens $\mathcal{O}(\log p)$ Berechnungen des Jacobi-Symbols.

3.18. (c) Sei $a$ die im vorigen Aufgabenteil berechnete Zahl. Die Elemente $a+1$ und -1 sind beides keine Quadrate, also ist $-(a+1)$ ein Quadrat. Man kann wegen $p \equiv$ $-1 \bmod 4$ effizient $b, c \in \mathbb{F}_{p}$ berechnen mit $b^{2}=a$ und $c^{2}=-(a+1)$. Nun gilt $b^{2}+c^{2}=-1$. Wir setzen $g=b+c X \in \mathbb{F}_{p^{2}}$.

Angenommen, $g$ ist ein Quadrat. Dann existieren $s, t \in \mathbb{F}_{p}$ mit $(s+t X)^{2}=g$. Dann ist $s^{2}-t^{2}=b$ und $2 s t=c$. Sei $h=(s-t X)^{2}$. Dann gilt $h=b-c X$ und damit $g h=b^{2}+c^{2}=-1$. Mit $r=(s+t X)(s-t X)=s^{2}+t^{2} \in \mathbb{F}_{p}$ gilt $r^{2}=g h=-1$. 
Dies ist ein Widerspruch zu $\left(\frac{-1}{p}\right)=-1$. Also ist $g$ kein Quadrat. Wir können nun den deterministischen Teil von Tonellis Algorithmus verwenden, um Wurzeln in $\mathbb{F}_{p^{2}} \mathrm{zu}$ ziehen.

3.19. Wir setzen $a=2$ und $t=0$ und beobachten, dass $\left(t^{2}-4 a\right)^{11} \equiv-1 \bmod 23$ gilt; also ist $t=0$ eine gültige Wahl für den ersten Schritt in Cipollas Algorithmus. Wir bestimmen nun den Grad von $X^{12}$ indem wir wiederholt $X^{2}$ durch $t X-a=-2$ ersetzen und die Koeffizienten in $\mathbb{Z} / 23 \mathbb{Z}$ berechnen:

$$
X^{12}=\left(X \cdot X^{2}\right)^{4} \equiv(-2 X)^{4}=2^{4}\left(X^{2}\right)^{2} \equiv 2^{4}(-2)^{2} \equiv 18 \bmod \left(X^{2}+2\right)
$$

Damit sind 18 und $5=23-18$ die Wurzeln von 2 in $\mathbb{F}_{23}$.

3.20. Der Beweis von Satz 3.12 verwendet nicht, dass $a$ ein Quadrat ist. Insbesondere ist $b \in \mathbb{K}$ stets eine Wurzel von $a \in \mathbb{F} \subseteq \mathbb{K}$. Wenn aber $a$ in $\mathbb{F}$ kein Quadat ist, dann muss $b \in \mathbb{K} \backslash \mathbb{E}$ gelten.

3.21. (a) Es gilt $\omega^{b / 2} \equiv-1 \bmod 97$ und $\omega^{b} \equiv 1 \bmod 97$.

3.21. (b) $F=\left(\begin{array}{cccc}1 & 1 & 1 & 1 \\ 1 & -22 & -1 & 22 \\ 1 & -1 & 1 & -1 \\ 1 & 22 & -1 & -22\end{array}\right) \quad \bar{F}=\left(\begin{array}{cccc}1 & 1 & 1 & 1 \\ 1 & 22 & -1 & -22 \\ 1 & -1 & 1 & -1 \\ 1 & -22 & -1 & 22\end{array}\right)$

3.21. (c) Der Grad von $f * g$ ist 3 , daher können wir $b=4$ wählen und $\omega=-22$ als primitive $b$-te Einheitswurzel verwenden. Es ergibt sich folgender Ablauf der schnellen Fourier-Transformation auf Eingabe von $f$ (links) bzw. $g$ (rechts):
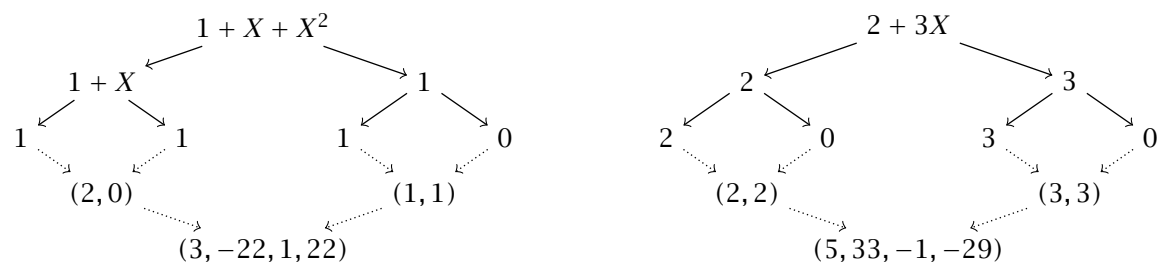

Dabei haben die Ablaufschemata folgende Bedeutung: Wenn $f, f_{0}, f_{1}$ Polynome sind, so dass $f(X)=f_{0}\left(X^{2}\right)+X f_{1}\left(X^{2}\right)$ gilt und $w=\left(1, \omega, \omega^{2}, \ldots, \omega^{b-1}\right)$ ist, dann symbolisiert ${ }_{f_{0}} \succ_{f_{1}}$ die Rekursion und ${ }_{(u, u)+w(v, v)}^{u v}$ die Zusammenführung der Ergebnisse, wobei die Addition und die Multiplikation komponentenweise gemeint sind. Wir berechnen nun komponentenweise das Produkt $(3,-22,1,22) \cdot(5,33,-1,-29) \equiv$ $(15,50,-1,41)$ mod 97 . Für die inverse Transformation benutzen wir das selbe Schema, nur benutzten wir die primitive $b$-te Einheitswurzel $\omega^{-1}=22$. Es ergibt sich folgender Ablauf: 


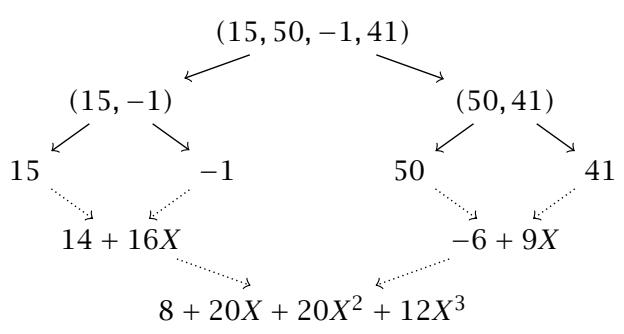

Um das Ergebnis zu erhalten, müssen wir $8+20 X+20 X^{2}+12 X^{3}$ noch mit $b^{-1}=-24$ multiplizieren und erhalten $(f * g)(X)=2+5 X+5 X^{2}+3 X^{3}$.

3.22. Seien $u, v$ natürliche Zahlen mit einer Binärdarstellung von höchstens $n$ Bits. Die Laufzeit des Verfahrens wird von der Laufzeit der schnellen Fourier-Transformation dominiert und ergibt sich daher $\mathrm{zu} \mathcal{O}(n \log n)$. Es gibt höchstens $m=\lceil n / 64\rceil$ viele Indizes $i$, bei denen sowohl $u_{i}$ als auch $v_{i}$ von Null verschieden sind. Wir betrachten das Produkt

$$
u v=\left(\sum_{j} u_{j} 2^{64 j}\right)\left(\sum_{j} v_{j} 2^{64 j}\right)=\sum_{j}\left(\sum_{k} u_{k} v_{j-k}\right) 2^{64 j}
$$

mit der üblichen Konvention $u_{j}=v_{j}=0$ für $j<0$ und für $j \geq m$. Damit sich $z_{j}=\sum_{k} u_{k} v_{j-k}$ eindeutig aus $w_{j}$ ergibt, muss $z_{j}<p_{1} p_{2} p_{3}$ gelten. Nun sind maximal $m$ viele Summanden in $z_{j}$ von Null verschieden, und jeder dieser Summanden ist durch $2^{64}$ beschränkt. Daher gilt $z_{j} \leq m \cdot 2^{64} \cdot 2^{64}$. Wegen $p_{i}>2^{56}$ genügt es, wenn für die Anzahl $m$ der 64-Bit-Blöcke von $u$ und $v$ gilt $m \leq 2^{3 \cdot 56} / 2^{2 \cdot 64}=2^{40}$. Weiterhin muss $2 m \leq 2^{56}$ gelten, damit bei der Fourier-Transformation kein Überlauf auftritt. Dies folgt bereits aus der Abschätzung $m \leq 2^{40}$. Damit können mit diesem Verfahren Zahlen mit einer Binärdarstellung $n$ mit $64 \cdot 2^{40}$ Bits multipliziert werden. Dies entspricht einer Binärdarstellung von mehr als acht Terabyte. Bei besonders großen Primzahlen $p_{i}$ (möglichst nahe an 64 Bits) lassen sich sogar noch größere Zahlen multiplizieren.

\section{Zu Kapitel 5}

5.1. Sei $p$ die Charakteristik von $K$. Ist $p \neq 2$, so ergänzen wir die linke Seite von Gleichung (5.2) quadratisch und erhalten $y^{2}+2\left(\frac{c}{2} x+\frac{d}{2}\right) y+\left(\frac{c}{2} x+\frac{d}{2}\right)^{2}-t(x)=$ $\left(y+\frac{c}{2} x+\frac{d}{2}\right)^{2}-t(x)$, wobei $t$ ein quadratisches Polynom in $x$ ist, welches wir auf die rechte Seite bringen. Nun setzen wir $y=y^{\prime \prime}+\frac{c}{2} x+\frac{d}{2}$ und $x^{\prime}=x^{\prime \prime}$ und fassen auf der rechten Seite die Koeffizienten zusammen. Starten wir mit Gleichung (5.3) und $p \neq 3$, so setzen wir $x^{\prime}=x-\frac{e^{\prime}}{3}$.

5.2. Wir rechnen in einem algebraisch abgeschlossenen Körperk, der $K$ als Unterkörper enthält. Hat das Polynom eine mehrfache Nullstelle, dann ist $x^{3}+A x+B=$ $(x-a)^{2}(x-b)$ mit $a, b \in k$. Ausmultiplizieren und Koeffizientenvergleich ergibt $2 a+b=0,2 a b+a^{2}=A$ und $-a^{2} b=B$. Setzt man $b=-2 a$ in die 
zweite und dritte Gleichung ein, so erhält man $A=-3 a^{2}$ und $B=2 a^{3}$. Es folgt $4 A^{3}+27 B^{2}=-4 \cdot 27 a^{6}+27 \cdot 4 a^{6}=0$. Dies gilt in jeder Charakteristik. Sei nun umgekehrt $4 A^{3}+27 B^{2}=0$. Ist die Charakteristik weder 2 noch 3, dann gibt es $a \in k$ mit $A=-3 a^{2}$ und $B=2 a^{3}$. Setzen wir $b=-2 a$, so liefert die gleiche Rechnung wie im ersten Teil $(x-a)^{2}(x-b)=x^{3}+A x+B$ und $a$ ist mehrfache Nullstelle. In Charakteristik 2 ist $4 A^{3}+27 B^{2}=0$ genau dann, wenn $B=0$ gilt. Dann ist $x^{3}+A x=x\left(x^{2}+A\right)=x(x+\mu)^{2}$ für ein geeignetes $\mu \in k$ (falls $A \in \mathbb{Z}$ ist, können wir einfach $\mu=A$ wählen). In Charakteristik 3 ist $4 A^{3}+27 B^{2}=0$ gleichbedeutend mit $A=0$. Wir erhalten dann $x^{3}+B=(x+v)^{3}$ für ein $v \in k$.

5.3. Wir setzen $s(x)=x^{3}+A x+B$. Ist $s(x)=0$, so gibt es genau den Punkt $(x, 0)$ auf $E\left(\mathbb{Z}_{p}\right)$. Hier ist $\left(\frac{f(x)}{p}\right)=0$. Dann ist $s(x)^{(p-1) / 2} \in\{1,-1\}$. Ist $f(x)^{(p-1) / 2}=1$, so ist $s(x)$ ein Quadrat in $\mathbb{F}_{p}$, und für $x$ haben wir die beiden Punkte $(x, y)$ und $(x,-y)$ auf $E\left(\mathbb{Z}_{p}\right)$. Mit anderen Worten, hier ist die Zahl der Punkte $\left(\frac{f(x)}{p}\right)+1=2$. Sei nun $s(x)^{(p-1) / 2}=-1$. Dann ist $s(x)$ kein Quadrat in $\mathbb{F}_{p}$, und für $x$ gibt es keinen Punkt $(x, y)$ auf $E\left(\mathbb{Z}_{p}\right)$. Hier ist die Zahl der Punkte $\left(\frac{f(x)}{p}\right)+1=0$.

5.4. (a) Wegen $4 \cdot 1^{3}+27 \cdot 6^{2}=3 \neq 0$ in $\mathbb{F}_{11}$ ist die Kurve elliptisch.

5.4. (b) Gehen wir systematisch vor, indem wir untersuchen, ob $x^{3}+x+6$ gleich 0 , ein Quadrat in $\mathbb{F}_{11}$ oder kein Quadrat in $\mathbb{F}_{11}$ ist, erhalten wir mit Aufgabe 5.3. die Menge $E\left(\mathbb{F}_{11}\right)=\{(2,4),(2,7),(3,5),(3,6),(5,2),(5,9),(7,2),(7,9)(8,3),(8,8),(10,2)$, $(10,9)\}$. Daher ist $\left|E\left(\mathbb{F}_{11}\right) \cup \mathcal{O}\right|=13$ eine Primzahl und $E\left(\mathbb{F}_{11}\right) \cup \mathcal{O}$ ist folglich zyklisch von der Ordnung 13.

5.5. (a) Wegen $4 \cdot 1^{3}+27 \cdot 1^{2}=1 \neq 0$ in $\mathbb{F}_{5}$ ist die Kurve elliptisch.

5.5. (b) Analog wie in der vorangegangenen Aufgabe erhalten wir $E\left(\mathbb{F}_{5}\right)=\{(0,0)$, $(2,0),(3,0)\}$. Jedes dieser drei Elemente hat Ordnung 2. Also gilt $E\left(\mathbb{F}_{5}\right) \cup \mathcal{O} \cong \mathbb{Z} / 2 \mathbb{Z} \times$ $\mathbb{Z} / 2 \mathbb{Z}$.

5.6. Sei $P=(x, y) \in E(k)$ ein Punkt der Ordnung 3, dann gilt $y \neq 0$, da $P$ sonst die Ordnung 2 hätte. Außerdem gilt $2 P=-P$ und es folgt $\left(\frac{3 x^{2}+A}{2 y}\right)^{2}-2 x=x$. Umformen und Einsetzen der Kurvengleichung ergibt $3 x^{4}+6 A x^{2}+12 B x-A^{2}=0$. Wir untersuchen jetzt das Polynom $t(x)=3 x^{4}+6 A x^{2}+12 B x-A^{2}$, welches unabhängig von der Existenz von $P$ definiert ist. Die Ableitung ist $t^{\prime}(x)=12\left(x^{3}+A x+B\right)$. Also hat $t^{\prime}$ keine mehrfachen Nullstellen, denn $x^{3}+A x+B$ hat bei elliptischen Kurven drei verschiedene Nullstellen. Daher hat $t$ keine dreifachen Nullstellen. Angenommen $t(x)$ hätte zwei verschiedene doppelte Nullstellen, dann gilt $t(x)=3(x-a)^{2}(x-b)^{2}=$ $3\left(x^{2}-2 a x+a^{2}\right)\left(x^{2}-2 b x+b^{2}\right)$ mit $a \neq b$. Ein Koeffizientenvergleich bei $x^{3}$ zeigt $0=-6(a+b)$. Hieraus folgt $a=-b$ und dann gilt $t(x)=3\left(x^{2}-a^{2}\right)^{2}=$ $3 x^{4}-6 a^{2} x^{2}+3 a^{4}$. Es folgt $B=0$ und $A=-a^{2}$ sowie $A^{2}=-3 a^{4}$. Dies impliziert $A=B=0$, was unmöglich ist. Also hat $t$ mindestens zwei einfache Nullstellen $a_{1} \neq a_{2}$. Wir wissen schon $a_{i}^{3}+A a_{i}+B \neq 0$ für $i=1$, 2. Damit finden wir für geeignete $b_{i} \neq 0$ vier verschiedene Punkte $\left(a_{i}, \pm b_{i}\right)$ auf $E(k)$. Wegen $t\left(a_{i}\right)=0$ und 
$b_{i}^{2}=a_{i}^{3}+A a_{i}+B \neq 0$ können wir rückwärts einsetzen und erhalten, dass alle vier Punkte die Ordnung 3 haben. Jede abelsche Gruppe, in der mindestens vier Elemente Ordnung 3 haben, enthält $\mathbb{Z} / 3 \mathbb{Z} \times \mathbb{Z} / 3 \mathbb{Z}$. Also gibt es mindestens 8 Punkte der Ordnung 3. Aber auf $E(k)$ kann es auch nicht mehr solche Punkte geben, denn deren $x$-Koordinaten sind Nullstellen von $t(x)$.

5.7. Für $\alpha=\beta$ gilt $(\alpha+\beta)(P)=2 \alpha(P)$, also ist in diesem Fall $\alpha+\beta$ rational, siehe die Bemerkung auf Seite 152. Als Nächstes betrachten wir den Fall $\alpha(P)=(f(P), g(P))$ und $\beta(P)=(f(P), h(P))$ für fast alle $P \in E(k)$. Wir können von $\alpha \neq \beta$ ausgehen, also ist $g(P) \neq h(P)$ für fast alle $P \in E(k)$. Damit bleibt nur $g(P)=-h(P)$ für unendlich viele $P \in E(k)$. Dies impliziert nun $g=-h$ in $k(x, y)$. Dann gilt schon $\alpha(P)=-\beta(P)$ für fast alle $P \in E(k)$. Also ist $\alpha=-\beta$ nach Lemma 5.13. Es bleibt der Fall $\alpha(P)=\left(f_{1}(P), g(P)\right)$ und $\beta(P)=\left(f_{2}(P), h(P)\right)$ für fast alle $P \in E(k)$ mit $f_{1}-f_{2} \neq 0 \in k(x, y)$. Für fast alle $P$ gilt also $f_{1}(P) \neq f_{2}(P)$ und wir finden über die Additionsformel auf der elliptischen Kurve für Punkte mit verschiedener $x$ Koordinate den entsprechenden rationalen Morphismus für $\alpha+\beta$.

5.8. Sei $\alpha(P)=\left(r_{1}(P), r_{2}(P)\right)$ für fast alle $P \in E(k)$. Wir wissen schon, dass wir $r_{i}(x, y)=\frac{u_{i}(x)+y v_{i}(x)}{u_{i}^{\prime}(x)+y v_{i}^{\prime}(x)}$ schreiben können. Nach einer Erweiterung mit dem Polynom $u_{i}^{\prime}(x)-y v_{i}(x)$ und einer Umbenennung erhalten wir $r_{i}(x, y)=\frac{u_{i}(x)+y v_{i}^{\prime}(x)}{q_{i}(x)}$. Jetzt benutzen wir, dass $\alpha$ ein Homomorphismus ist. Also ist $\alpha(x,-y)=-\alpha(x, y)$. Hieraus folgt $v_{1}^{\prime}(x)=0 \in k[x]$ und $u_{2}(x)=0 \in k[x]$. Nach einer weiteren Umbenennung erhalten wir die vier Polynome $p(x), q(x), u(x), v(x) \in k[x]$. Wäre $p(x) / q(x)$ oder $u(x) / v(x)$ konstant, so wäre das Bild von $\alpha$ eine endliche Menge. Dies widerspricht der Endlichkeit des Kerns.

5.9. $\mathrm{Zu}$ zeigen ist nur, dass $\phi_{q}$ ein Gruppenhomomorphismus ist. Da $\phi_{q}$ eine Bijektion von $E(k)$ ist, gibt es die Umkehrabbildung $\psi(P)=\phi_{q}^{-1}(P)$, und es reicht zu zeigen, dass $\psi$ ein Gruppenhomomorphismus ist. Wir identifizieren $E(k) \cup\{\mathcal{O}\}$ mit $\operatorname{Pic}^{0}(E(k))$ entsprechend Satz 5.5. Die Abbildung $P \mapsto \psi(P)$ induziert einen Automorphismus der Divisorengruppe, den wir wieder $\psi$ nennen. $\mathrm{Zu}$ zeigen ist jetzt, dass Hauptdivisoren auf Hauptdivisoren geschickt werden, denn dann induziert $\psi$ einen Homomorphismus auf $\operatorname{Pic}^{0}(E(k))$. Betrachte einen Hauptdivisor $\operatorname{div}(f)=\sum_{P \in E(k)}$ $\operatorname{ord}_{P}(f) P$ mit $f \in k[x, y]$. Es folgt

$$
\psi(\operatorname{div}(f))=\sum_{P \in E(k)} \operatorname{ord}_{P}(f) \psi(P)=\sum_{P \in E(k)} \operatorname{ord}_{\phi_{q}(P)}(f) P
$$

Aus Satz 5.3 folgt $\operatorname{ord}_{\phi_{q}(P)}(f)=q \cdot \operatorname{ord}_{P}(f)=\operatorname{ord}_{P}\left(f^{q}\right)$ für alle $P \in E(k)$. Also ist $\psi(\operatorname{div}(f))=\operatorname{div}\left(f^{q}\right)=q \cdot \operatorname{div}(f)$ ein Hauptdivisor. Damit induziert $\psi$ einen Automorphismus der abelschen Gruppe $E(k) \cup\{\mathcal{O}\}$. Die Umkehrabbildung $\phi_{q}$ ist damit ebenfalls ein Homomorphismus.

5.10. Wir schreiben $\alpha$ als rationalen Morphismus als $\alpha=(f(x, y), g(x, y))$ mit $f(x, y)=\left(\frac{3 x^{2}+A}{2 y}\right)^{2}-2 x$. Für ein geeignetes Polynom $p(x)$ finden wir $f(x, y)=$ 
$\frac{p(x)}{s(x)}$ mit $s(x)=x^{3}+A x+B$. Die Nullstellen von $s(x)$ sind einfach, insbesondere verschwindet $s^{\prime}(x)$ nicht identisch, und für $y=0$ muss bei $\frac{p(x)}{s(x)}$ ein Pol vorliegen. Daher haben $p(x)$ und $s(x)$ keine gemeinsamen Nullstellen.

5.11. Der Frobenius-Morphismus $\phi_{q}$ ist ein Endomorphismus, also auch $\left(\phi_{q}-1\right)$ nach Satz 5.15. (Dies kann auch direkt gezeigt werden.) Der Kern besteht (neben $\mathcal{O}$ ) genau aus den Punkten $(a, b) \in E(k)$ mit $a=a^{q}$ und $b=b^{q}$. Dies heißt nichts anderes als $(a, b) \in E\left(\mathbb{F}_{q}\right)$. (Siehe etwa den Beweis von Satz 1.58.)

\section{Zu Kapitel 6}

6.1. Wir schreiben $u \sim v$, wenn $u$ und $v$ transponiert sind. Es gilt $a b c=a(b c) \sim$ $(b c) a=b a c=(b a) c \sim c(b a)=c b a$, aber $a b c+c b a$.

6.2. Für die Richtung von (i) nach (ii) können wir $M=\Sigma^{*}$ annehmen. Sei $\varphi(a)=1$ für alle $a \in \Sigma$. Dann definiert $\varphi: \Sigma^{*} \rightarrow \mathbb{N}$ einen Homomorphismus mit $\varphi(w)=0$ genau dann, wenn $w$ leer ist (die Zahl $\varphi(w)$ ist die Länge von $w$ ). Das leere Wort ist das neutrale Element von $\Sigma^{*}$. Wenn $p q=x y$ gilt, dann schreiben wir $p=a_{1} \cdots a_{k}$, $q=a_{k+1} \cdots a_{m}, x=b_{1} \cdots b_{\ell}, y=b_{\ell+1} \cdots b_{n}$ mit $a_{i}, b_{j} \in \Sigma$. Aus $p q=x y$ folgt $m=n$ und $a_{i}=b_{i}$ für alle $i \in\{1, \ldots, n\}$. Ohne Einschränkung sei $k \geq \ell$, andernfalls vertauschen wir $(p, q)$ und $(x, y)$. Mit $u=a_{\ell+1} \cdots a_{k}$ gilt also $p=x u$ und $y=u q$.

Sei nun $M$ ein Monoid, welches (ii) erfüllt. Mit $\tilde{M}=M \backslash\{1\}$ setzen wir $\Sigma=$ $\tilde{M} \backslash\{a b \mid a, b \in \tilde{M}\}$. Die Menge $\Sigma$ enthält die unzerlegbaren Elemente aus $M$. Mit Induktion nach $\varphi(w)$ zeigen wir, dass sich jedes Element aus $M$ als Produkt von Elementen aus $\Sigma$ schreiben lässt. Wenn $\varphi(w)=0$, dann ist $w$ das neutrale Element; und dieses ergibt sich als das leere Produkt. Sei nun $\varphi(w)>0$. Wenn $w \in \Sigma$ gilt, dann ist nichts weiter zu zeigen. Sei also $w=u v$ mit $u, v \in \tilde{M}$. Dann gilt $\varphi(u)<$ $\varphi(u)+\varphi(v)=\varphi(w)$. Analog ist $\varphi(v)<\varphi(w)$. Mit Induktion lassen sich $u$ und $v$ als Produkte von Elementen aus $\Sigma$ schreiben und damit auch $w=u v$. Dies zeigt, dass $M$ von $\Sigma$ erzeugt wird.

Sei nun $a_{1} \cdots a_{m}=b_{1} \cdots b_{n}$ mit $a_{i}, b_{j} \in \Sigma$. Um zu zeigen, dass $M$ dem freien Monoid $\Sigma^{*}$ entspricht, müssen wir $m=n$ und $a_{i}=b_{i}$ für alle $i \in\{1, \ldots, m\}$ nachweisen. Dies geschieht mit Induktion nach $m+n$. Für $m=0$ ist $0=\varphi(1)=$ $\varphi\left(b_{1} \cdots b_{n}\right)=\varphi\left(b_{1}\right)+\cdots+\varphi\left(b_{n}\right)$. Aus $\varphi(c)>0$ für alle $c \in \Sigma$ folgt $n=0$. Sei nun $m \geq 1$. Wegen $\varphi\left(b_{1} \cdots b_{n}\right)=\varphi\left(a_{1} \cdots a_{m}\right)>0$ gilt $n \geq 1$. Dann existiert $u \in M$ mit $a_{1}=b_{1} u, b_{2} \cdots b_{n}=u a_{2} \cdots a_{m}$ oder $b_{1}=a_{1} u, a_{2} \cdots a_{m}=$ $u b_{2} \cdots b_{n}$. Ohne Einschränkung sei $a_{1}=b_{1} u$ und $b_{2} \cdots b_{n}=u a_{2} \cdots a_{m}$. Aus $a_{1}, b_{1} \in \Sigma$ und der Konstruktion von $\Sigma$ folgt $u=1$; dies zeigt $a_{1}=b_{1}$ und $a_{2} \cdots a_{m}$ $=b_{2} \cdots b_{n}$. Mit Induktion folgt $m=n$ und $a_{i}=b_{i}$ für alle $i \in\{2, \ldots, m\}$.

6.3. Betrachte eine beliebige nicht triviale Gruppe $G$. Dann ist $G$ kein freies Monoid. Für $p q=x y$ gibt es $u \in G$ mit $p u=x$. Hieraus folgt $y=u q$. 
6.4. (a) Sei $u \prec v$. Ist $u$ echter Präfix von $v$, so ist auch $w u$ echter Präfix von $w v$. Ist $u=r a s$ und $v=r b t$ mit $r, s, t \in \Sigma^{*}, a, b \in \Sigma$ und $a<b$, so ist $w u=(w r) a s$ und $w v=(w r) b t$. In beiden Fällen ergibt sich also $w u \prec w v$. Sei umgekehrt $w u \prec w v$. Ist $w u$ ein echter Präfix von $w v$, so ist auch $u$ ein echter Präfix von $v$. Ist $w u=r^{\prime}$ as und $w v=r^{\prime} b t$ und $a<b$ so ist $|w| \leq\left|r^{\prime}\right|$. Sei $r^{\prime}=w r$. Dann ist $u=r a s$ und $v=r b t$. Es ergibt sich also $u \prec v$.

6.4. (b) Da $u$ kein Präfix von $v$ ist muss $u=r a s$ und $v=r b t \operatorname{mit} r, s, t \in \Sigma^{*}, a, b \in$ $\Sigma$ und $a<b$ gelten. Es ergibt sich $u w=r a(s w)$ und $v z=r b(t z)$ und damit $u w \prec v z$.

6.5. (i) $\Rightarrow$ (ii): Angenommen, $w$ ist ein echter Faktor von $w^{2}$, also $w^{2}=u w v$ mit $u \neq \varepsilon \neq v$. Dann gibt es Wörter $s, t \in \Sigma^{*}$ mit $u w=w t$ und $w v=s w$. Damit ergibt sich $|u|=|t|,|v|=|s|$ und $w=s t=u s=t v$. Daraus erhalten wir $u=t$ und $s=v$, woraus $w=s t=u s=t s$ folgt. Nach Satz 6.3 ist $w$ also nicht primitiv. (ii) $\Rightarrow$ (i): Ist $w=u^{i}$ mit $i>1$ so ergibt sich $w^{2}=u^{2 i}=u\left(u^{i}\right) u^{i-1}$ und $w$ ist ein echter Faktor von $w^{2}$. (i) $\Leftrightarrow$ (iii) gilt, denn $w=u^{i}$ mit $i>1, u=a u^{\prime}$ gilt genau dann wenn $v a=u^{\prime}\left(a u^{\prime}\right)^{i-1} a=\left(u^{\prime} a\right)^{i}$ ist. Die Wurzel $u^{\prime} a$ von $v a$ ist also die zyklische Vertauschung der Wurzel $u$ von $w$.

6.6. Seien $u$ und $v$ Primitivwurzeln. Dann gilt ohne Einschränkung $w=u^{i}=v^{j}$ mit $1 \leq i \leq j$. Für $i=1$ ist $w$ primitiv und daher $u=v=w$. Für $i \geq 2$ gilt $|u|+|v| \leq$ $|w|$ und nach dem Korollar 6.5 (Satz von Fine und Wilf) ist auch $\operatorname{ggT}(|u|,|v|)$ eine Periode von $w$. Dies bedeutet $u=v$.

6.7. (i) $\Rightarrow$ (ii): Sei $v$ ein echter Suffix von $w=u v$. Wir zeigen zunächst, dass $v$ kein echter Präfix von $w$ sein kann. Dazu nehmen wir an, dass $w=v t$ gilt. Nach Satz 6.3 ist $v=(r s)^{k} r, u=r s$ und $t=s r$ für $r, s \in \Sigma^{*}$. Weil $w$ primitiv ist, kann man $r \neq \varepsilon$ und $r s \neq s r$ annehmen. Da $w$ ein Lyndon-Wort ist, gilt $w=(r s)^{k+1} r \prec r(r s)^{k+1}$. Wegen Aufgabe 6.4. kann man das Wort $r$ vorne kürzen und es ergibt sich $(s r)^{k+1} \prec$ $(r s)^{k+1}$. Wieder nach Aufgabe 6.4. kann man dies mit $r$ von rechts multiplizieren und es ergibt sich $(s r)^{k+1} r \prec(r s)^{k+1} r=w$. Dies ist ein Widerspruch dazu, dass $w$ ein Lyndon-Wort ist. Also sind echte Suffixe von Lyndon-Wörtern keine Präfixe. Falls nun $v \prec u v=w$ gilt, so ist nach Aufgabe 6.4. $v u \prec u v$. Dies ist nicht möglich, da $w$ ein Lyndon-Wort ist. Also gilt $w \prec v$ wie gefordert. (ii) $\Rightarrow$ (i): Für Faktorisierungen $w=u v$ gilt $u v \prec v \prec v u$. Ist $w=u^{i}$ mit $i>1$ so ist $w \prec u \prec u^{i}=w$. Also ist $w$ primitiv. Damit ist $w$ ein Lyndon-Wort. Im Rest der Lösung verwenden wir die Äquivalenz (i) $\Leftrightarrow$ (ii). (i) $\Rightarrow$ (iii): Die Aussage ist klar für $w \in \Sigma$. Sei also $w \notin \Sigma$. Da alle Buchstaben auch Lyndon-Wörter sind, kann man $w=u v$ setzen mit $|v|$ maximal, so dass $u \neq \varepsilon$ gilt und $v$ ein Lyndon-Wort ist. Es gilt $u \prec u v \prec v$, da $u v$ ein Lyndon-Wort ist. Also bleibt zu zeigen, dass $u$ ebenfalls ein Lyndon-Wort ist. Sei ohne Einschränkung $u \notin \Sigma$, sonst ist $u$ bereits ein Lyndon-Wort. Wir betrachten nun einen echten Suffix $u^{\prime}$ von $u$. Das Wort $u^{\prime} v$ ist kein Lyndon-Wort, da $v$ maximal gewählt wurde. Also gibt es einen Suffix $t$ von $u^{\prime} v$ mit $t \prec u^{\prime} v$. Aus $u^{\prime} \prec t$ folgt 
$u^{\prime} \prec t \prec u^{\prime} v$ und damit $t=u^{\prime} s$. Das Wort $s$ ist ein Suffix des Lyndon-Wortes $v$, und somit gilt $v \prec s$ was $u^{\prime} v \prec u^{\prime} s=t$ impliziert, ein Widerspruch. Damit gilt $t \preceq u^{\prime}$ und wir erhalten zusammen $u \prec u v \prec t \preceq u^{\prime}$. Mit (ii) folgt, dass $u$ ein LyndonWort ist, was zu zeigen war. (iii) $\Rightarrow$ (i): Falls $w \in \Sigma$, so ist die Aussage klar. Also ist $w=u v$ mit $u \prec v$, wobei $u, v$ Lyndon-Wörter sind. Wir zeigen zunächst $u v \prec v$ als Hilfsaussage. Ist $u$ kein Präfix von $v$, so gilt dies nach Aufgabe 6.4. Ist $v=u v^{\prime}$, so ist $v \prec v^{\prime}$, weil $v$ ein Lyndon-Wort ist. Damit gilt $u v \prec u v^{\prime}=v$. Wir zeigen nun $u v \prec s$ für jeden echten Suffix $s$ von $u v$. Ist $s$ ein Suffix von $v$, so gilt $u v \prec v \prec s$. Sonst gilt $s=t v$. Da $t$ ein echter Suffix von $u$ ist, gilt $u \prec t$ und damit $u v \prec t v=s$ nach Aufgabe 6.4. Also ist $u v$ ein Lyndon-Wort.

6.8. Da jeder Buchstabe aus $\Sigma$ ein Lyndon-Wort ist, existiert eine Zerlegung von $w$ in Lyndon-Wörter. Sei $w=\ell_{1} \cdots \ell_{n}$ eine solche Zerlegung, wobei $n$ minimal ist. Falls $\ell_{i} \prec \ell_{i+1}$ an einer Stelle $i \in\{1, \ldots, n-1\}$ gilt, so ist $\ell_{i} \ell_{i+1}$ nach Aufgabe 6.7. ein Lyndon-Wort. Dies kann nicht sein, da $n$ minimal ist. Also gilt $\ell_{n} \preceq \ell_{n-1} \preceq \ldots \preceq \ell_{1}$. Damit gibt es eine Zerlegung der geforderten Art. Es verbleibt die Eindeutigkeit dieser Zerlegung zu zeigen. Seien also $w=\ell_{1} \cdots \ell_{n}=\ell_{1}^{\prime} \cdots \ell_{m}^{\prime}$ zwei solche Zerlegungen. Wir zeigen $\ell_{1}=\ell_{1}^{\prime}$ und folgern die Aussage dann per Induktion auf dem Wort $\ell_{2} \cdots \ell_{n}=\ell_{2}^{\prime} \cdots \ell_{m}^{\prime}$. Sei also ohne Einschränkung $\ell_{1}=\ell_{1}^{\prime} \cdots \ell_{i}^{\prime} v$, wobei $i \geq 1$ gilt und $v$ ein nichtleerer Präfix von $\ell_{i+1}^{\prime}$ ist. Dann ist $\ell_{1} \prec v$ nach Aufgabe 6.7. Außerdem gilt $v \preceq \ell_{i+1}^{\prime} \preceq \ell_{1}^{\prime} \prec \ell_{1}$, da $v$ ein Präfix von $\ell_{i+1}^{\prime}$ ist. Zusammen gilt also $\ell_{1} \prec \ell_{1}$, ein Widerspruch.

\section{Zu Kapitel 7}

7.1. (a) Da $S$ endlich ist, existieren $t, p \geq 1$ mit $x^{t}=x^{t+p}$. Es folgt $x^{j}=x^{j+\ell p}$ für alle $j \geq t$ und $\ell \geq 0$.

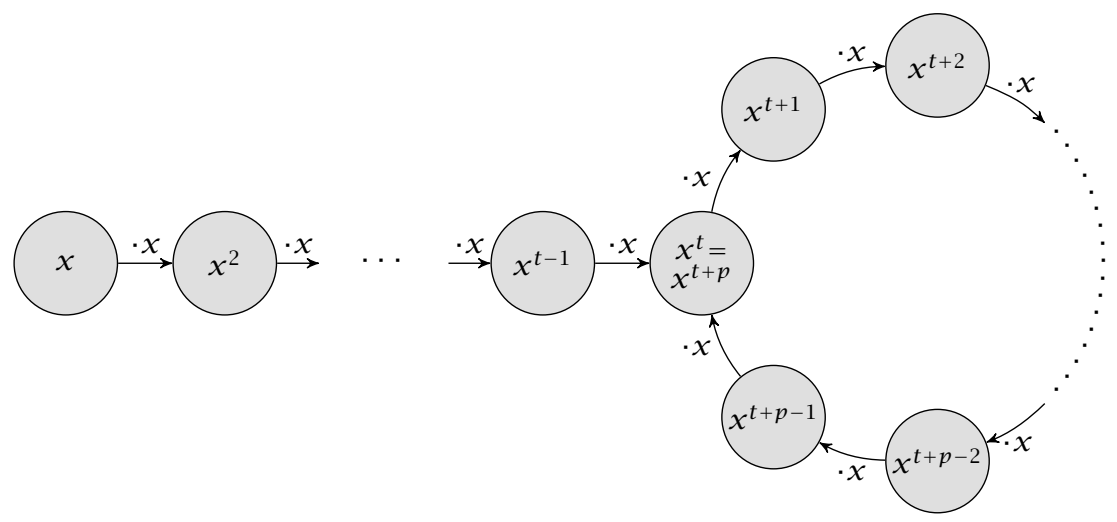


Also gilt $x^{2 t p}=x^{t p+t p}=x^{t p}$, und $x^{t p}$ ist idempotent. Seien $x^{m}$ und $x^{n}$ idempotent. Dann gilt $x^{n}=\left(x^{n}\right)^{2}=\left(x^{n}\right)^{3}=\cdots$ und damit $x^{m}=\left(x^{m}\right)^{n}=x^{m n}=$ $\left(x^{n}\right)^{m}=x^{n}$. Dies zeigt, dass $\left\{x^{k} \mid k \geq 1\right\}$ genau ein Idempotentes enthält.

7.1. (b) Es gilt $\left\{x^{k} \mid k \geq 1\right\}=\left\{x^{k}|1 \leq k \leq| S \mid\right\}$, da sich die Element spätestens ab dem Exponenten $k=|S|+1$ wiederholen. Also existiert für alle $x \in S$ eine Zahl $n(x) \in\{1, \ldots,|S|\}$, so dass $x^{n(x)}$ idempotent ist. Dann gilt $x^{|S| !}=x^{n(x) \cdot m^{\prime}}=$ $\left(x^{n(x)}\right)^{m^{\prime}}=\left(x^{2 n(x)}\right)^{m^{\prime}}=x^{2 n(x) \cdot m^{\prime}}=x^{2|S| !}$ für $|S| !=n(x) \cdot m^{\prime}$. Also ist $x^{|S| !}$ idempotent.

7.2. Wie wir in der Lösung von Aufgabe 7.1. (a) gesehen haben, gibt es Zahlen $t, p \in$ $\mathbb{N}$ mit $p \geq 1$ und $x^{t}=x^{t+p}$. Wenn wir $t$ und $p$ minimal wählen, dann ist $S=$ $\left\{x, \ldots, x^{t+p-1}\right\}$ und $n=t+p-1$. Insbesondere ist die Halbgruppe eindeutig durch $t \in\{1, \ldots, n\}$ gegeben, und unterschiedliche Werte für $t$ definieren nichtisomorphe Halbgruppen.

7.3. Man kann auf $2^{M} \operatorname{durch} A \cdot B=\{a b \in M \mid a \in A, b \in B\}$ für $A, B \subseteq M$ eine assoziative Verknüpfung definieren. Also existiert eine Zahl $n$ mit $A^{n}=A^{2 n}$ für alle $A \subseteq M$. Insbesondere gilt $\varphi\left(\Sigma^{n}\right)=\varphi(\Sigma)^{n}=\varphi(\Sigma)^{2 n}=\varphi\left(\Sigma^{2 n}\right)$.

7.4. Sei $\varphi: M \rightarrow N$ ein Homomorphismus in ein endliches Monoid $N$, welcher $L$ erkennt. Setze $P=\left\{x \in N \mid x^{2} \in \varphi(L)\right\}$. Dann gilt

$$
\begin{aligned}
\varphi^{-1}(P) & =\{u \in M \mid \varphi(u) \in P\}=\{u \in M \mid \varphi(u u)=\varphi(u) \varphi(u) \in \varphi(L)\} \\
& =\{u \in M \mid u u \in L\}=\sqrt{L}
\end{aligned}
$$

Also gilt $\varphi^{-1}(\varphi(\sqrt{L}))=\varphi^{-1}\left(\varphi\left(\varphi^{-1}(P)\right)\right)=\varphi^{-1}(P)=\sqrt{L}$, und $\sqrt{L}$ wird von $N$ erkannt.

7.5. Sei zunächst $L=K_{1} \times K_{2}$, und für $i \in\{1,2\}$ sei $\varphi_{i}: M_{i} \rightarrow N_{i}$ ein Homomorphismus in ein endliches Monoid $N_{i}$ mit $K_{i}=\varphi_{i}^{-1}\left(\varphi_{i}\left(K_{i}\right)\right)$. Sei $\psi: M_{1} \times M_{2} \rightarrow N_{1} \times N_{2}$ mit $\psi\left(m_{1}, m_{2}\right)=\left(\varphi_{1}\left(m_{1}\right), \varphi_{2}\left(m_{2}\right)\right)$. Dann gilt

$$
\begin{aligned}
\psi^{-1}(\psi(L)) & =\psi^{-1}\left(\varphi_{1}\left(K_{1}\right) \times \varphi_{2}\left(K_{2}\right)\right) \\
& =\varphi_{1}^{-1}\left(\varphi_{1}\left(K_{1}\right)\right) \times \varphi_{2}^{-1}\left(\varphi_{2}\left(K_{2}\right)\right)=K_{1} \times K_{2}=L
\end{aligned}
$$

Also ist $L$ erkennbar. Die Richtung von rechts nach links folgt nun, weil erkennbare Sprachen abgeschlossen sind unter Vereinigung.

Für die Umkehrung sei $\varphi: M_{1} \times M_{2} \rightarrow N$ ein Homomorphismus in ein endliches Monoid $N$ mit $\varphi^{-1}(\varphi(L))=L$. Für $i \in\{1,2\}$ definieren wir die Homomorphismen $\psi_{1}: M_{1} \rightarrow N$ und $\psi_{2}: M_{2} \rightarrow N$ durch $\psi_{1}\left(m_{1}\right)=\varphi\left(m_{1}, 1\right)$ und $\psi_{2}\left(m_{2}\right)=\varphi\left(1, m_{2}\right)$. Dies liefert den Homomorphismus $\psi: M_{1} \times M_{2} \rightarrow N \times N$ mit $\psi\left(m_{1}, m_{2}\right)=\left(\psi_{1}\left(m_{1}\right), \psi_{2}\left(m_{2}\right)\right)$. Wir zeigen nun, dass auch der Homomorphismus $\psi$ die Sprache $L$ erkennt. Sei hierzu:

$$
P=\left\{\left(n_{1}, n_{2}\right) \in N \times N \mid n_{1} n_{2} \in \varphi(L)\right\}
$$


Dann gilt:

$$
\begin{aligned}
\psi^{-1}(P) & =\left\{\left(m_{1}, m_{2}\right) \mid \psi\left(m_{1}, m_{2}\right) \in P\right\} \\
& =\left\{\left(m_{1}, m_{2}\right) \mid \psi_{1}\left(m_{1}\right) \psi_{2}\left(m_{2}\right) \in \varphi(L)\right\} \\
& =\left\{\left(m_{1}, m_{2}\right) \mid \varphi\left(m_{1}, 1\right) \varphi\left(1, m_{2}\right) \in \varphi(L)\right\} \\
& =\left\{\left(m_{1}, m_{2}\right) \mid \varphi\left(m_{1}, m_{2}\right) \in \varphi(L)\right\}=\varphi^{-1}(\varphi(L))=L
\end{aligned}
$$

Es folgt $\psi^{-1}(\psi(L))=\psi^{-1}\left(\psi\left(\psi^{-1}(P)\right)\right)=\psi^{-1}(P)=L$. Also erkennt $\psi$ die Menge L. Damit ist

$$
L=\psi^{-1}(\psi(L))=\bigcup_{\left(n_{1}, n_{2}\right) \in \psi(L)} \psi^{-1}\left(n_{1}, n_{2}\right)=\bigcup_{\left(n_{1}, n_{2}\right) \in \psi(L)} \psi_{1}^{-1}\left(n_{1}\right) \times \psi_{2}^{-1}\left(n_{2}\right)
$$

und $L$ hat die gewünschte Form.

7.6. Es ist $u \in L(\mathcal{B}) \Leftrightarrow q_{0} \cdot u \in Q \backslash F \Leftrightarrow q_{0} \cdot u \notin F \Leftrightarrow u \in M \backslash L(\mathcal{A})$.

7.7. (a) Wir wählen $F=F_{1} \times Q_{2} \cup Q_{1} \times F_{2}$. Dann ist $u \in L(\mathcal{B})$ genau dann wenn $\left(q_{1}, q_{2}\right) \cdot u \in F$. Dies ist äquivalent zu $q_{1} \cdot u \in F_{1}$ oder $q_{2} \cdot u \in F_{2}$, also zu $u \in$ $L\left(\mathcal{A}_{1}\right) \cup L\left(\mathcal{A}_{2}\right)$.

7.7. (b) Wir wählen $F=F_{1} \times F_{2}$. Dann ist $u \in L(\mathcal{B})$ genau dann wenn $\left(q_{1}, q_{2}\right) \cdot u \in F$. Dies ist äquivalent zu $q_{1} \cdot u \in F_{1}$ und $q_{2} \cdot u \in F_{2}$, also zu $u \in L\left(\mathcal{A}_{1}\right) \cap L\left(\mathcal{A}_{2}\right)$.

7.8. (a) Wir wenden die Thompson-Konstruktion aus Lemma 7.12 an. Bei dem entstehenden Automaten eliminieren wir Zustände, welche nur zwischen zwei $\varepsilon$-Transitionen stehen.

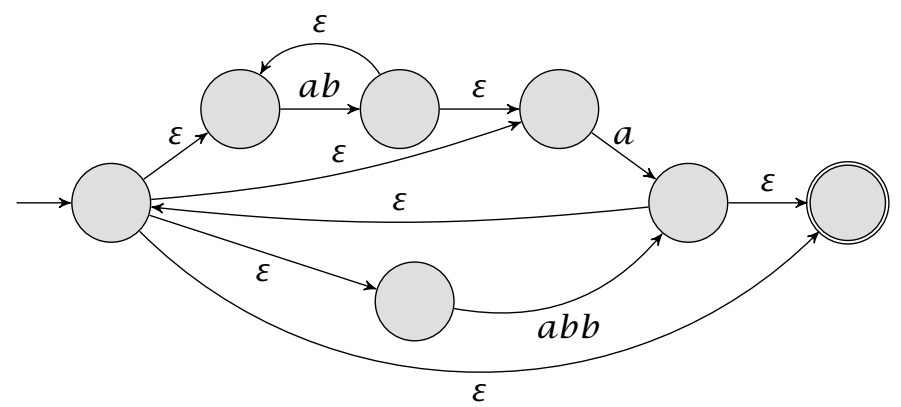

7.8. (b) Die Konstruktion aus Lemma 7.13 liefert den folgenden buchstabierenden Automaten. 


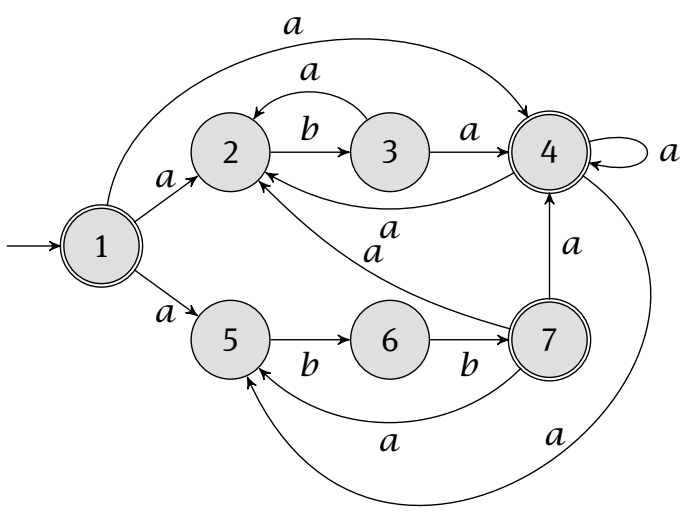

7.8. (c) Wir verwenden die Potenzautomatenkonstruktion aus Satz 7.18. Den Zustand $\varnothing$ haben wir der besseren Übersicht halber nicht dargestellt.

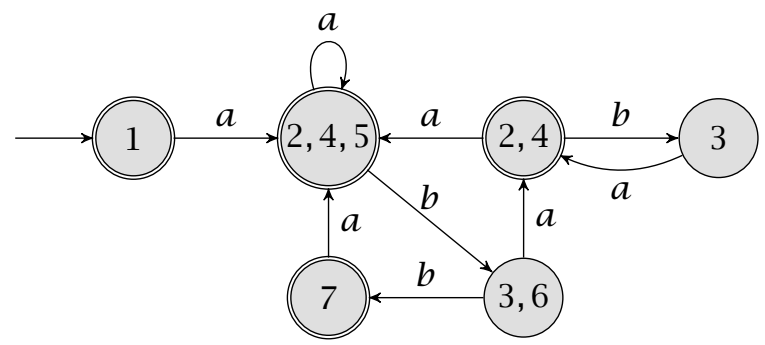

7.8. (d) Wir können die Zustände $\{2,4,5\}$ und $\{2,4\}$ zu einem Endzustand und die Zustände $\{3\}$ und $\{3,6\}$ zu einem Zustand verschmelzen. Es ergibt sich der folgende Automat. Dabei wird für den nächsten Schritt der Automat gleich vollständig angegeben, d. h., es wird ein Fangzustand eingeführt, in den alle undefinierten Übergänge gehen.

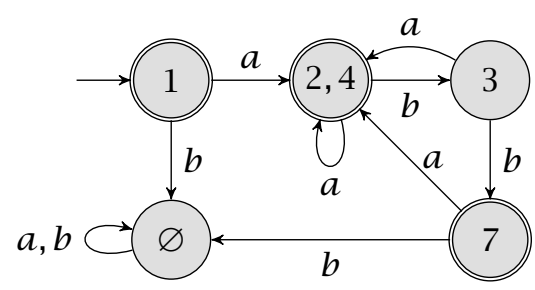

7.8. (e) Es genügt, bei $\mathcal{B}^{\prime}$ die Endzustände und die Nicht-Endzustände zu vertauschen. 


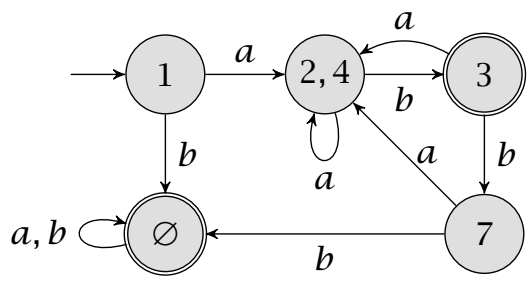

7.8. (f) Wir benutzen die Konstruktion aus Lemma 7.14 um einen rationalen Ausdruck zu erhalten. Da es mehrere Endzustände gibt, wird ein neuer Endzustand eingefügt und die alten Endzustände mit $\varepsilon$-Kanten damit verbunden.

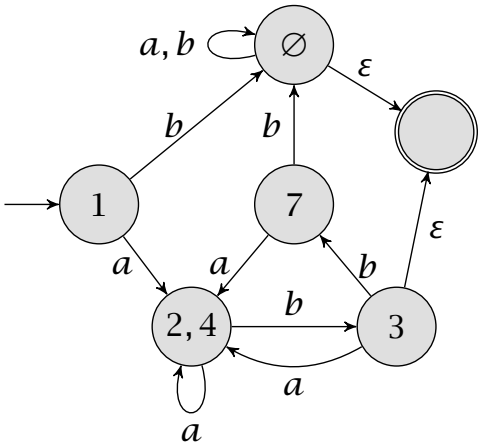

(a) Ursprungsgraph mit $\varepsilon$-Kanten

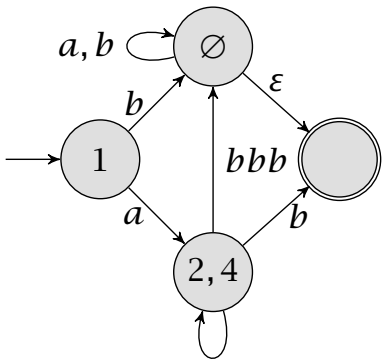

$a, b a, b b a$

(c) Entfernen von Knoten $\{7\}$

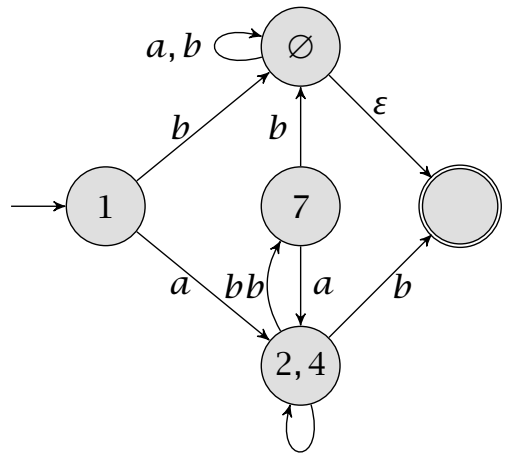

$a, b a$

(b) Entfernen von Knoten $\{3\}$

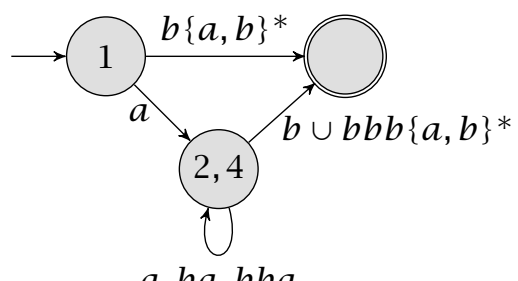

(d) Entfernen von Knoten $\varnothing$

Im letzten Schritt wird nun der Knoten $\{2,4\}$ entfernt und es ergibt sich $\Sigma^{*} \backslash L=$ $b\{a, b\}^{*} \cup a\{a, b a, b b a\}^{*}\left(b \cup b b b\{a, b\}^{*}\right)$.

7.9. Zustände in $\mathcal{P}\left(\mathcal{A}^{\rho}\right)$ sind Teilmengen von $Q$. Für diesen Automaten gilt $P \cdot a=$ $\{q \in Q \mid \exists p \in P:(q, a, p) \in \delta\}$. Ferner ist $F$ der Startzustand und $P \subseteq Q$ ist genau dann ein Endzustand, wenn $q_{0} \in P$ gilt. Wegen $L\left(\mathcal{P}\left(\mathcal{A}^{\rho}\right)\right)=L(\mathcal{A})^{\rho}$ ist nur zu zei- 
gen, dass verschiedene Zustände in $\mathcal{P}\left(\mathcal{A}^{\rho}\right)$ eine verschiedene Leistung haben. Sei also $P \neq P^{\prime}$ und ohne Einschränkung $q \in P \backslash P^{\prime}$. Nach Annahme gibt es ein Wort $w=a_{1} \cdots a_{n}$ mit $a_{i} \in \Sigma$ und $q_{0} \cdot a_{1} \cdots a_{n}=q$. Damit gehört $w^{\rho}=a_{n} \cdots a_{1}$ zur Leistung von $P$. Da $\mathcal{A}$ deterministisch ist, gehört jedoch $w^{\rho}$ nur zur Leistung von Zuständen in $\mathcal{P}\left(\mathcal{A}^{\rho}\right)$, die $q$ enthalten. Insbesondere gehört $w^{\rho}$ nicht zur Leistung von $P^{\prime}$.

7.10. (a) Die Aussage folgt mit der Konstruktion im Beweis von Satz 7.4 zusammen mit dem Abschluss von V unter direkten Produkten.

7.10. (b) Das Monoid Synt $(L)$ erkennt die Sprache $L$. Da V abgeschlossen ist unter Divisoren, folgt die Aussage schließlich aus Satz 7.4.

7.11. Es sei $\left(2^{\Sigma}, \cup, \varnothing\right)$ das Monoid der Teilmengen von $\Sigma$ und alph : $\Sigma^{*} \rightarrow 2^{\Sigma}$ der kanonische Homomorphismus, der durch $a \mapsto\{a\}$ definiert ist. Dann ist alph surjektiv und alph erkennt jede Sprache $L$, die eine boolesche Kombination der Form $B^{*}$ für $B \subseteq \sum$ ist. In $2^{\Sigma}$ gelten die Gleichungen $x^{2}=x$ sowie $x y=y x$. Das syntaktische Monoid von $L$ ist ein homomorphes Bild von $2^{\Sigma}$, also gelten dort ebenfalls die Gleichungen $x^{2}=x$ sowie $x y=y x$.

Sei nun $\varphi: \Sigma^{*} \rightarrow M$ ein $L$ erkennender Homomorphismus in ein Monoid, in dem die Gleichungen $x^{2}=x$ und $x y=y x$ gelten. Sei $u=a_{1} \cdots a_{n}$ ein Wort mit $a_{i} \in \Sigma$. Aufgrund der Gleichungen in $M$ sehen wir $\phi(u)=\prod_{a \in \operatorname{alph}(u)} \phi(a)$. Hieraus folgt, dass $\operatorname{alph}(u)$ den Wert $\phi(u)$ bestimmt. Mit der Schreibweise $[A]=$ $\left\{u \in \Sigma^{*} \mid \operatorname{alph}(u)=A\right\}$ gilt

$$
L=\bigcup_{A \in \operatorname{alph}(L)}[A]=\bigcup_{A \in \operatorname{alph}(L)} A^{*} \backslash\left(\bigcup_{B \subsetneq A} B^{*}\right)
$$

Also hat $L$ die gewünschte Form. Man kann noch bemerken, dass keine Endlichkeit von $M$ benutzt wurde. Das Bild $\phi\left(\Sigma^{*}\right)$ ist allein aufgrund der Gleichungen endlich.

7.12. (a) Divisoren von aperiodischen Monoiden sind aperiodisch. Für die Richtung von links nach rechts genügt es also, eine aperiodische Gruppe $G$ zu betrachten. In $G$ gilt $1=g^{n}\left(g^{-1}\right)^{n}=g^{n+1}\left(g^{-1}\right)^{n}=g \cdot 1=g$ für ein $n \in \mathbb{N}$. Also besteht die Gruppe $G$ nur aus dem neutralen Element.

Seien nun alle Gruppendivisoren von $M$ trivial. Wir setzen $n=|M|$ !. Nach Aufgabe 7.1. (b) gilt $x^{n}=x^{2 n}$ für alle $x \in M$. Für jedes $x \in M$ ist die Menge $U=$ $\{1\} \cup\left\{x^{m} \mid m \geq n\right\}$ ein Untermonoid von $M$. Die Abbildung $\varphi: U \rightarrow\left\{x^{m} \mid m \geq n\right\}$ mit $\varphi(1)=x^{n}$ und $\varphi\left(x^{m}\right)=x^{m}$ definiert einen surjektiven Homomorphismus, $\mathrm{da}$ $x^{m} \cdot x^{n}=x^{n} \cdot x^{m}=x^{n} \cdot x^{n+m^{\prime}}=x^{n} x^{n} x^{m^{\prime}}=x^{2 n} x^{m^{\prime}}=x^{n} x^{m^{\prime}}=x^{m}$ gilt. Außerdem ist $G=\left\{x^{m} \mid m \geq n\right\}$ eine Gruppe mit neutralem Element $x^{n}$. Das Inverse von $x^{m}$ ist $x^{(n-1) m}$, da $x^{m} \cdot x^{(n-1) m}=x^{n m}=x^{n}$ gilt. Nach Voraussetzung ist $\left|\left\{x^{m} \mid m \geq n\right\}\right|=1$ und damit $x^{n}=x^{n+1}$.

7.12. (b) Wenn $M$ aperiodisch ist, dann gilt $M \in C$ nach dem Krohn-Rhodes-Theorem und der vorigen Teilaufgabe. Für die Umkehrung bemerken wir, dass $U_{2}$ aperiodisch 
ist, und dass Divisoren von aperiodischen Monoiden aperiodisch sind. Es verbleibt zu zeigen, dass das Kranzprodukt von aperiodischen Monoiden aperiodisch ist. Seien $M, N$ zwei aperiodische Monoide mit $x^{n}=x^{n+1}$ für alle $x \in M \cup N$. Wir betrachten nun das Kranzprodukt $M \succ N$ und zeigen $(f, x)^{2 n}=(f, x)^{2 n+1}$ für alle $(f, x) \in$ $M^{N} \times N$. Es gilt

$$
(f, x)^{2 n}=\left(f * x f * x^{2} f * \cdots * x^{2 n-1} f, x^{2 n}\right)
$$

Mit $x^{n}=x^{m}$ für alle $m \geq n$ folgt

$$
\begin{aligned}
(f, x)^{2 n} & =\left(f * x f * \cdots * x^{n} f * x^{n} f * \cdots * x^{n} f, x^{2 n}\right) \\
& =\left(f * x f * \cdots * x^{n-1} f *\left(x^{n} f\right)^{n}, x^{2 n}\right)
\end{aligned}
$$

In $M^{N}$ gilt $\left(x^{n} f\right)^{n}=\left(x^{n} f\right)^{n+1}$, und in $N$ gilt $x^{2 n}=x^{2 n+1}$. Also ist

$$
(f, x)^{2 n}=\left(f * x f * \cdots * x^{n-1} f *\left(x^{n} f\right)^{n+1}, x^{2 n+1}\right)=(f, x)^{2 n+1}
$$

Dies zeigt, dass $M \succ N$ aperiodisch ist.

7.13. Für

$$
\begin{aligned}
J & =\bigcup\left\{[s][t]^{\omega} \mid s, t \in S,[s][t]^{\omega} \cap L \neq \varnothing\right\} \\
K & =\bigcup\left\{[s][e]^{\omega} \mid s, e \in S, s e=s, e^{2}=e,[s][e]^{\omega} \cap L \neq \varnothing\right\}
\end{aligned}
$$

gilt $K \subseteq J$. Zu zeigen ist, dass $L=J$ genau dann gilt, wenn $L=K$ ist. Hierfür weisen wir $J \subseteq K$ nach. Wir betrachten $s^{\prime}, t \in S$ mit $\left[s^{\prime}\right][t]^{\omega} \cap L \neq \varnothing$. Sei $e=t^{n}$ das von $t$ erzeugte Idempotente (welches nach Aufgabe 7.1. (a) existiert), und sei $s=s^{\prime} e$. Nun ist $e^{2}=e$ und $s e=s^{\prime} e e=s^{\prime} e=s$, und es gilt $\left[s^{\prime}\right][t]^{\omega} \subseteq[s][e]^{\omega}$. Die letzte Eigenschaft sieht man wie folgt: jedes Wort $\alpha \in\left[s^{\prime}\right][t]^{\omega}$ lässt sich schreiben als $\alpha=u_{0} u_{1} \cdots$ mit $\varphi\left(u_{0}\right)=s^{\prime}$ und $\varphi\left(u_{i}\right)=t$ für $i \geq 1$; nun gilt $\varphi\left(u_{0} \cdots u_{n}\right)=s$ und $\varphi\left(u_{i+1} \cdots u_{i+n}\right)=e$, so dass $\alpha \in[s][e]^{\omega}$ ist. Es folgt $[s][e]^{\omega} \cap L \neq \varnothing$ und $[s][e]^{\omega} \subseteq K$. Dies zeigt $\left[s^{\prime}\right][t]^{\omega} \subseteq K$ und $J \subseteq K$.

7.14. Wir nehmen zunächst an, dass $\varphi$ die Sprache $L$ erkennt. Sei $\alpha \sim_{\varphi} \beta$ und $\alpha \in L$ mit $\alpha=u_{1} u_{2} \cdots, \beta=v_{1} v_{2} \cdots$ und $\varphi\left(u_{i}\right)=x_{i}=\varphi\left(v_{i}\right)$. Wir wollen $\beta \in$ $L$ zeigen. Nach Lemma 7.44 existiert $t \in S$ und eine Folge von Indizes $1 \leq i_{1}<$ $i_{2}<\cdots$ mit $x_{i_{j}+1} \cdots x_{i_{j+1}}=t$ für alle $j \geq 1$. Insbesondere gilt für die Wörter $u_{j}^{\prime}=u_{i_{j}+1} \cdots u_{i_{j+1}}$ und $v_{j}^{\prime}=v_{i_{j}+1} \cdots v_{i_{j+1}}$, dass $\varphi\left(u_{j}^{\prime}\right)=\varphi\left(v_{j}^{\prime}\right)=t$. Sei $u_{0}^{\prime}=$ $u_{1} \cdots u_{i_{1}}$ und $v_{0}^{\prime}=v_{1} \cdots v_{i_{1}}$. Dann ist $\varphi\left(u_{0}^{\prime}\right)=\varphi\left(v_{0}^{\prime}\right)=s$ für ein $s \in S$, und es gilt $\alpha=u_{0}^{\prime} u_{1}^{\prime} \cdots$ sowie $\beta=v_{0}^{\prime} v_{1}^{\prime} \cdots$. Aus $\alpha \in[s][t]^{\omega} \cap L$ folgt $[s][t]^{\omega} \subseteq L$, und mit $\beta \in[s][t]^{\omega}$ erhalten wir schließlich $\beta \in L$.

Betrachte $\alpha \in[s][t]^{\omega} \cap L$ und $\beta \in[s][t]^{\omega}$. Dann gilt $\alpha \sim_{\varphi} \beta$ aufgrund der Faktorisierung, welche sich aus $\alpha, \beta \in[s][t]^{\omega}$ ergibt. Daraus folgt $\beta \in L$ und $[s][t]^{\omega} \subseteq L$. 


\section{Zu Kapitel 8}

8.1. Die Idee ist, zunächst $w \underset{S}{\stackrel{*}{\Longrightarrow}} \widehat{w} \in \operatorname{IRR}(S)$ und $z \stackrel{*}{\Rightarrow} \hat{z} \in \operatorname{IRR}(S)$ zu berechnen und danach die irreduziblen Wörter $\widehat{w}$ und $\hat{z}$ zeichenweise zu vergleichen. Es reicht daher, einen Algorithmus anzugeben, der auf eine Eingabe $w \in \Sigma^{*}$ in der Zeit $\mathcal{O}(|w|)$ die Berechnung $w \underset{S}{\stackrel{*}{\Longrightarrow}} \widehat{w} \in \operatorname{IRR}(S)$ durchführt.

Wir wählen ein $\delta>0$ mit $|\ell| \geq(1+\delta)|r|$ für alle $(\ell, r) \in S$. Dies ist möglich, da $S$ endlich und längenverkürzend ist. Dann geben wir einem Wortpaar $(u, v)$ das Gewicht $\gamma(u, v)=|u|+(1+\delta)|v|$. Wir beginnen mit dem Wortpaar $(1, w)$, wobei 1 das leere Wort sei. Setze $n=|w|$. Dann gilt $\gamma(1, w)=(1+\delta) n$ und $1 \in \operatorname{IRR}(S)$. Wir behalten als eine Invariante, dass wir nur Wortpaare $(u, v)$ erzeugen mit $u \in \operatorname{IRR}(S)$ und $u v=w$ in $M$, also $u v \stackrel{*}{\Longleftrightarrow} w$. Ziel ist, das Wortpaar $(\widehat{w}, 1)$ nach linear vielen Schritten zu erhalten. Ist dies noch nicht erreicht, so gilt $v=a v^{\prime}$ für ein $a \in \Sigma$ und $v^{\prime} \in \Sigma^{*}$. Ist nun $u a \in \operatorname{IRR}(S)$, also $u a$ irreduzibel, so ersetzen wir das $\operatorname{Paar}(u, v)$ in einem Zeitschritt durch das Paar $\left(u a, v^{\prime}\right)$. Das Gewicht hat dabei um die Konstante $\delta$ abgenommen. Sei also $u a$ reduzibel, dann gilt notwendigerweise $u a=u^{\prime} \ell$ für ein $(\ell, r) \in S$. Wir ersetzen in diesem Fall $(u, v)$ in einem Zeitschritt durch das Paar $\left(u^{\prime}, r v^{\prime}\right)$. Für die Gewichte gilt $\gamma\left(u^{\prime}, r v^{\prime}\right) \leq \gamma\left(u^{\prime} \ell, v^{\prime}\right)=\gamma\left(u a, v^{\prime}\right) \leq \gamma(u, v)-$ $\delta$. Offensichtlich wird in beiden Fällen die Invariante erhalten. der Algorithmus ist also korrekt und berechnet $\widehat{w}$ in der Zeit $\left(1+\frac{1}{\delta}\right) n \in \mathcal{O}(n)$.

8.2. (a) Das System $S_{+}$terminiert, da es längenlexikographisch reduzierend ist für die durch $I_{+}$induzierte Ordnung auf $\Sigma$. Es ist lokal konfluent, denn für $c a b \underset{S_{+}}{\stackrel{c}{\leftrightarrows} b a} \underset{S_{+}}{\Longrightarrow}$ $b c a$ gilt $c a b \underset{S_{+}}{\Longrightarrow} a c b \underset{S_{+}}{\Longrightarrow} a b c \underset{S_{+}}{\Longleftarrow} b a c \underset{S_{+}}{\Longleftarrow} b c a$.

8.2. (b) Für alle Paare $(a, b) \in I$ muss entweder $b a \rightarrow a b \in S$ oder $a b \rightarrow b a \in S$ gelten. Setze $I_{+}=\{(a, b) \in \Sigma \times \Sigma \mid b a \rightarrow a b \in S\}$. Dann ist zunächst $I=\{(a, b)$, $\left.(b, a) \mid(a, b) \in I_{+}\right\}$. Angenommen, $I_{+}$wäre keine transitive Orientierung. Dann gibt es $(a, b),(b, c) \in I_{+}$und $(a, c) \notin I_{+}$. Also enthält $S$ Regeln $b a \rightarrow a b$ und $c b \rightarrow b c$, aber keine Regel $c a \rightarrow a c$. Betrachte für $n \in \mathbb{N}$ die Ersetzungen $c^{n} a^{n} b^{n} \stackrel{*}{\stackrel{S_{+}}{\leftrightarrows}} c^{n} b^{n} a^{n}$ $\stackrel{*}{\Rightarrow} b^{n} c^{n} a^{n}$. Die Faktoren $a^{n} b^{n}$ und $b^{n} c^{n}$ sind irreduzibel, denn wäre etwa ein anderes Wort in der Klasse von $a^{n} b^{n}$ irreduzibel, so müsste in diesem Wort irgendwo ein Faktor $b a$ erscheinen. Dies ist aber nicht möglich. Nun ist auch $c^{n} a^{n}$ irreduzibel, denn entweder ist $(a, c) \notin I$ oder $a c \rightarrow c a \in S$. Da $S$ endlich ist, gilt notwendigerweise $c^{n} a^{n} b^{n}, b^{n} c^{n} a^{n} \in \operatorname{IRR}(S)$ sofern $n$ genügend groß ist, im Widerspruch zu $c^{n} a^{n} b^{n}=b^{n} c^{n} a^{n} \in M\left(\sum, I\right)$.

8.2. (c) Es sei $M=M(\Sigma, I)$, dann können wir jedem Element aus $M$ vermöge (8.1) eine Länge zuordnen. Daher wird $M$ von den Elementen der Länge 1 erzeugt und diese Erzeugendenmenge ist minimal. Es gilt nun ist $\Sigma=(M \backslash\{1\}) \backslash(M \backslash\{1\})^{2}$ und damit ist $\Sigma$ durch $M$ bestimmt. Die Kantenmenge $I$ ergibt sich durch die Elemente aus $\Sigma$, die verschieden sind, aber in $M$ kommutieren. 
8.2. (d) Nach Aufgabe 8.2. (c) gilt $\Sigma=\{a, b, c\}$ und $I=\{(a, c),(c, a)\}$ mit $a c=c a$ sowie $a b \neq b a$ und $b c \neq c b$ in $M$. Dann sind $a b c$ und $c a b$ transponiert und es sind auch $c a b=a c b$ und $c b a$ transponiert. Aber es gibt keine Transposition von $a b c$, die direkt zu $c b a$ führt.

8.2. (e) Alle Graphen mit vier Knoten haben eine transitive Orientierung und der Kreis $C_{5}$ mit fünf Knoten besitzt keine. Die Antwort lautet also „5“.

8.3. (a) Wir wählen eine lineare Ordnung für $\Sigma$. Das System ist dann fast identisch zu dem System aus Gleichung (8.2) bzw. 8.3. Wir müssen nur zusätzlich alle Quadrate von Buchstaben löschen.

$$
\begin{aligned}
S_{\text {RACC }}= & \{b u a \rightarrow a b u \mid a, b \in \Sigma, a<b,(a, b u) \in I\} \\
& \cup\{a u a \rightarrow u \mid a \in \Sigma,(a, u) \in I\}
\end{aligned}
$$

8.3. (b) Es sei $\tilde{V}$ eine disjunkte Kopie von $V$ und $\Sigma=V \cup \tilde{V}$. Für $(a, b) \in E$ nehmen wir $(a, b),(\tilde{a}, b),(a, \tilde{b}),(\tilde{a}, \tilde{b})$ in $I$ auf, aber keine weiteren Paare. Insbesondere kommutieren $a$ und $\tilde{a}$ nicht in $C$. Betrachte jetzt den durch $a \mapsto a \tilde{a}$ induzierten Homomorphismus $\phi: G \rightarrow C$. (Beachte, $(a, b) \in E$ impliziert $a \tilde{a} b \tilde{b}=b \tilde{b} a \tilde{a}$ in $C$.) $\mathrm{Zu}$ zeigen bleibt, dass $\phi$ injektiv ist. Hierfür wählen wir zunächst eine lineare Ordnung für $\Sigma$, in der die Elemente $a$ und $\tilde{a}$ jeweils direkt nebeneinander angeordnet sind. Wegen $V \subseteq \Sigma$ induziert dies auch eine lineare Ordnung auf $\Sigma$. Sei jetzt $1 \neq g \in G$ und $w \in V^{*}$ ein längenlexikographisch kürzestes Wort, welches $g$ repräsentiert. Dann ist $\phi(w)$ eine irreduzible Normalformen für das System $S_{\text {RACC }}$ aus der Lösung zu 8.3. (a), denn die Faktoren $a \tilde{a}$ und $\tilde{a} a$ verhindern, dass identische Buchstaben nebeneinander stehen.

8.3. (c) Die Konvergenz von $T$ ist reine Routine. Wir zeigen nur, dass $\phi: \Sigma \rightarrow \mathcal{F}, a \rightarrow$ $\{a\}$ einen Isomorphismus $\phi: C(\Sigma, I) \rightarrow \mathcal{F}^{*} / T$ induziert. Für $(a, b) \in I$ erhalten wir $\phi(a) \phi(b)=\{a, b\}$ in $\mathcal{F}^{*} / T$. Ferner gilt $\phi\left(a^{2}\right)=\varnothing=1$ in $\mathcal{F}^{*} / T$ für $a \in \Sigma$. Da $\phi(\Sigma)$ die Gruppe $\mathcal{F}^{*} / T$ erzeugt, ist $\phi$ surjektiv. Betrachte jetzt die Abbildung $\psi$ : $\mathcal{F} \rightarrow C(\Sigma, I), F \rightarrow \prod_{a \in F} a$. Da $F \in \mathcal{F}$ eine Clique ist, ist es unerheblich, in welcher Reihenfolge wir das Produkt $\prod_{a \in F} a \in C(\Sigma, I)$ auswerten. Aufgrund der Regeln in $T$ induziert $\psi$ einen Homomorphismus von $\mathcal{F}^{*} / T$ auf $C(\Sigma, I)$. Wegen $\psi(\phi(a))=a$ für alle $a \in \Sigma$ sind $\phi$ und $\psi$ invers zueinander. Insbesondere ist $\phi$ ein Isomorphismus.

8.4. Seien $G_{1}$ und $G_{2}$ zwei endlich erzeugte Untergruppen einer freien Gruppe $F$. Ohne Einschränkung ist $F=F(\Sigma)$ eine endlich erzeugte freie Gruppe. Nach Abschnitt 8.6 sind die Mengen der frei reduzierten Wörter in $\left(\Sigma \cup \Sigma^{-1}\right)^{*}$, die die Elemente $G_{1}$ und $G_{2}$ darstellen, jeweils regulär. Der Durchschnitt regulärer Sprachen ist regulär; und dies liefert eine rationale Menge für den Durchschnitt $G_{1} \cap G_{2}$.

8.5. Sei $K$ der Kern der Projektion $F_{2} \rightarrow \mathbb{Z}, a \mapsto 1, b \mapsto 0$. Der Schreiergraph von $K$ hat als Knotenmenge $\left\{K a^{n} \mid n \in \mathbb{Z}\right\}$ und jeder Knoten $K a^{n}$ hat eine ausgehende Kante, 
die mit $a$ (bzw. $a^{-1}$ ) beschriftet ist, zu $K a^{n+1}$ (bzw. $K a^{n-1}$ ), und eine Schlinge mit Beschriftung $b$ und $b^{-1}$.

Die mit $a, a^{-1}$ beschrifteten Kanten bilden einen Spannbaum. Sei $\Delta$ die Menge der Kanten, die mit $b$ beschriftet sind. Nach Satz 8.15 ist $K$ isomorph zur freien Gruppe $F(\Delta)$. Im Beweis dieses Satzes wird ein Isomorphismus definiert. Dieser liefert genau die Menge $U$ als Bild von $\Delta$.

8.6. Sei $G$ eine von $k$ Elementen erzeugte Gruppe und $\phi: G \rightarrow G$ ein surjektiver Homomorphismus. Angenommen, $\phi$ wäre nicht injektiv. Dann gibt es ein $1 \neq g \in G$ mit $\phi(g)=1$. Da $G$ residuell endlich ist, existiert ein surjektiver Homomorphismus $\pi: G \rightarrow E$ auf eine endliche Gruppe $E$ mit $\pi(g) \neq 1$. Betrachte für $n \in \mathbb{N}$ jetzt den Homomorphismus $\pi_{n}: G \rightarrow E$, der durch $\pi_{n}(h)=\pi\left(\phi^{n}(h)\right)$ definiert ist. Da $\phi$ surjektiv ist, finden wir ein $h_{n} \in G$ mit $\phi^{n}\left(h_{n}\right)=g$. Damit gilt $\pi_{n}\left(h_{n}\right)=\pi(g) \neq 1$ aber $\pi_{n+1}\left(h_{n}\right)=\pi(\phi(g))=\pi(1)=1$. Daher ist $\pi_{m}\left(h_{n}\right)=1$ für alle $m>n$. Insbesondere gilt $\pi_{m} \neq \pi_{n}$ für alle $m \neq n$. Ein Homomorphismus von $G$ nach $E$ ist durch die Bilder der $k$ Erzeugenden bestimmt. Also gibt es höchstens $|E|^{k}$ Homomorphismen. Dies ist ein Widerspruch und $\phi$ muss injektiv sein.

8.7. Wir zeigen, dass $\mathrm{BS}(p, q)$ nicht hopfsch ist, wenn $p$ und $q$ nicht dieselbe Menge an Primteilern haben. Sei $r$ eine Primzahl, die $p$, aber nicht $q$ teilt. Im umgekehrten Fall verwendet man den Isomorphismus $\operatorname{BS}(p, q) \rightarrow \operatorname{BS}(q, p)$, gegeben durch $a \mapsto a, t \mapsto t^{-1}$. Wir betrachten das Element $\left[a, t a^{p / r} t^{-1}\right]$. Hierbei steht $[x, y]$ für $x y x^{-1} y^{-1}$, den sogenannten Kommutator von $x$ und $y$. Er ist genau dann 1, wenn $x y=y x$. Das Wort $\left[a, t a^{p / r} t^{-1}\right]=a t a^{p / r} t^{-1} a^{-1} t a^{-p / r} t^{-1}$ ist Britton-reduziert, also insbesondere nicht das Einselement. Es liegt aber im Kern des Homomorphismus $\phi: \operatorname{BS}(p, q) \rightarrow \operatorname{BS}(p, q)$ mit $t \mapsto t$ und $a \mapsto a^{r}$. Der Homomorphismus $\phi$ ist demzufolge nicht injektiv. Die Abbildung $\phi$ ist aber surjektiv, da $r$ und $q$ teilerfremd sind und $a$ sich deswegen als Produkt von Elementen $\phi(a)=a^{r}$ und $\phi\left(t a^{p / r} t^{-1}\right)=a^{q}$ darstellen lässt. Daher ist $\mathrm{BS}(p, q)$ unter den genannten Voraussetzungen nicht hopfsch.

8.8. In der Gruppe BG $(1,2)=\mathrm{BS}(1,2) * F(b) /\left\{b a b^{-1}=t\right\}$ gilt $b a^{e} b^{-1}=t^{e}$ und $t^{e} a t^{-e}=a^{2^{e}}$. Setzen wir $A(0)=a$ und $A(n+1)=b A(n) b^{-1} a b A(n)^{-1} b^{-1}$, so hat $A(n)$ nur exponentielle Länge, aber die Normalform von $A(n)$ hat die Gestalt $a^{T(n)}$.

8.9. Es seien $x, y \in F$ mit $x y=y x$, wobei $F$ frei ist. Wir können annehmen, dass $x$ und $y$ frei reduzierte Wörter sind. Ist $x y$ ebenfalls frei reduziert, so gilt dies auch für $y x$ und die Behauptung folgt aus Satz 6.3 (b). Ohne Einschränkung sei $|x|>|y|$ und wir können $x=x^{\prime} s$ und $y=s^{-1} y^{\prime}$ für einen maximalen Suffix $s$ von $x$ schreiben, der nicht leer ist. Für $y=s^{-1}$ gilt $x y=x^{\prime}=y x=y x^{\prime} y^{-1}$, also $x^{\prime} y=y x^{\prime}$ und die Behauptung folgt mit Induktion nach $|x|$. Daher gilt ohne Einschränkung auch, dass $y^{\prime}$ ein nichtleeres Wort ist. Die mit $s$ konjugierten Elemente $s x^{\prime}=s x s^{-1}$ und $y^{\prime} s^{-1}=s y s^{-1}$. kommutieren. Ist $s x^{\prime}$ nicht frei reduziert, so können wir Induktion nach $|x y|$ benutzen und sind fertig. Also ist $s x^{\prime}$ frei reduziert. Aufgrund der maxi- 
malen Länge von $s$ und der Symmetrie in $x$ und $y$ ist jetzt jedoch das Wort $s x^{\prime} y^{\prime} s$ frei reduziert; und wir können erneut Satz 6.3 (b) anwenden.

8.10. (a) Es gilt $\rho_{b \bar{a}}=i_{b} \circ \lambda_{a b} \circ i_{b}$. Es reicht daher zu zeigen, dass jeder WhiteheadAutomorphismus durch Automorphismen der Form $i_{a}, \lambda_{a b}$ und $\rho_{b \bar{a}}$ ausgedrückt werden kann. Betrachte hierfür zunächst eine Permutation $\pi_{a b}$ von $\Sigma$, die zwei Buchstaben $a, b \in \sum a \neq b$ vertauscht und alle anderen Buchstaben invariant lässt. Es gilt $\pi_{a b}=\lambda_{b a}^{-1} \circ i_{a} \circ \rho_{b \bar{a}} \circ \lambda_{b a}$, denn $\rho_{b \bar{a}} \circ \lambda_{b a}(a)=b$ und $\lambda_{b a}^{-1} \circ i_{a}(b)=b$ sowie $\rho_{b \bar{a}} \circ \lambda_{b a}(b)=b a^{-1}$ und $\lambda_{b a}^{-1} \circ i_{a}\left(b a^{-1}\right)=a$. Daher lassen sich alle Transpositionen darstellen, die wiederum die volle Permutationsgruppe erzeugen. Es verbleibt, die Whitehead-Automorphismen der Form $W_{(a, L, R, M)}$ darzustellen. Dies ist möglich wegen $W_{(a, L, R, M)}=\prod_{b \in L \cup M} \lambda_{a b} \cdot \prod_{c \in R \cup M} \rho_{c \bar{a}}$.

8.10. (b) Wir können $|\Sigma| \geq 2$ annehmen. Die Permutationsgruppe von $\Sigma$ wird von einer Transposition und einer zyklischen Vertauschung erzeugt. Für $|\Sigma|=2$ reicht eine Transposition. Wir benötigen jetzt nur noch die Automorphismen $i_{a}$ und $\lambda_{a b}$ für ein einziges Paar $(a, b)$ mit $a, b \in \Sigma$ und $a \neq b$, um zunächst alle regulären und elementaren Nielsen-Transformationen zu erzeugen. Nach Teilaufgabe 8.10. (a) erzeugen diese dann die volle Automorphismengruppe.

8.11. Die Lösung ergibt sich per Induktion nach $\ell$, indem $\operatorname{man} \min \left\{a_{1}, a_{3}\right\}, \min \left\{a_{2}\right.$, $\left.a_{4}\right\} \leq F_{\ell}$ und $\max \left\{a_{1}, a_{3}\right\}, \max \left\{a_{2}, a_{4}\right\} \leq F_{\ell+1}$ zeigt.

8.12. (a) Sei $A \in \operatorname{PSL}(2, \mathbb{Z})$ mit der Wirkung $A(z)=\frac{a z+b}{c z+d}$ ein Element der Ordnung 2 . Dann haben $A$ und $A^{2}$ die Matrixdarstellungen:

$$
A=\left(\begin{array}{ll}
a & b \\
c & d
\end{array}\right) \quad A^{2}=\left(\begin{array}{cc}
a^{2}+b c & b(a+d) \\
c(a+d) & d^{2}+b c
\end{array}\right)
$$

Wäre $b=c=0$, so wäre $a d=1$, also $a=d= \pm 1$ und $A(z)=z$ für alle $z$, im Widerspruch dazu, dass $A$ Ordnung 2 hat. Also ist $b \neq 0$ oder $c \neq 0$ und es folgt $a+d=0$.

8.12. (b) Seien $S(z)=\frac{-1}{z}$ und $R(z)=\frac{-1}{z+1}$ wie im Text gewählt. Nach einer Konjugation mit $R$ oder $R^{2}$ können wir wie in dem Beweis von Satz 8.28 davon ausgehen, dass $A$ eine Darstellung der Form $R^{i_{1}} S \cdots R^{i_{m-1}} S R^{i_{m}}$ mit $m \geq 2$ und $1 \leq i_{\ell} \leq 2$ für alle $1 \leq \ell \leq m$ hat. (Die Werte $m=0,1$ sind ausgeschlossen, da $A$ die Ordnung 2 hat.) Ohne Einschränkung gilt $i_{1}=1$ und $i_{m}=2$, denn für $i_{1}+i_{m} \neq 3$ hat $A$ eine unendliche Ordnung. Für $m=2$ ist dann $A=R S R^{2}$ konjugiert zu $S$. Also gilt $m \geq 3$; und wir erhalten nach einer Konjugation $S R^{2} A R S=R^{i_{2}} S \cdots R^{i_{m-2}} S R^{i_{m-1}}$. Die Behauptung folgt mit Induktion nach $m$.

8.13. (a) Sei -1 quadratischer Rest modulo $n$. Es gibt also $p, q \in \mathbb{Z}$ mit $-q^{2}-p n=1$. Wir bilden $A \in \operatorname{PSL}(2, \mathbb{Z})$ mit $A(z)=\frac{q z+n}{p z-q}$. Dann hat $A$ Ordnung 2. Nach 8.12. (b) ist $A$ in $\operatorname{PSL}(2, \mathbb{Z})$ konjugiert zu $S$, d. h., es gibt ein $X \in \operatorname{PSL}(2, \mathbb{Z}) \operatorname{mit} X(z)=\frac{x z+y}{u z+v}$ und 
$X S X^{-1}=A$. Es folgt

$$
X S X^{-1}(z)=\frac{(-v y-u x) z+x^{2}+y^{2}}{\left(-v^{2}-u^{2}\right) z+v y+u x}=\frac{q z+n}{p z-q}
$$

Wegen $n \in \mathbb{N}$ ist also $n=x^{2}+y^{2}$.

8.13. (b) Nach Vorraussetzung ist $\operatorname{ggT}(x, y)=1$, also gibt es $u, v \in \mathbb{Z}$ mit $x v$ $y u=1$. Wir bilden $X \in \operatorname{PSL}(2, \mathbb{Z})$ mit $X(z)=\frac{x z+y}{u z+v}$. Es folgt wie eben $X S X^{-1}(z)=$ $\frac{q z+x^{2}+y^{2}}{p z-q}=\frac{q z+n}{p z-q}$ für $p=-v^{2}-u^{2}$ und $q=-v y-u x$. Aus $-q^{2}-p n=1$ folgt $-1 \equiv q^{2} \bmod n$.

8.13. (c) Der Fall $p=2$ ist trivial. Sei nun $p \geq 3$. Für $p=x^{2}+y^{2} \operatorname{gilt} \operatorname{ggT}(x, y)=1$. Nach 8.13. (a) und 8.13. (b) ist jetzt $p$ genau dann die Summer zweier Quadrate, wenn -1 quadratischer Rest modulo $p$ ist; und -1 ist genau dann ein quadratischer Rest modulo $p$, wenn $(\mathbb{Z} / p \mathbb{Z})^{*}$ ein Element der Ordnung 4 hat. Die multiplikative Gruppe $(\mathbb{Z} / p \mathbb{Z})^{*}$ ist zyklisch und hat die Ordnung $p-1$. Sie enthält genau dann ein Element der Ordnung 4, wenn $p \equiv 1 \bmod 4$ gilt. 
